\title{
DLG2 impairs dsDNA break repair and maintains genome integrity in neuroblastoma
}

\author{
Simon Keane ${ }^{\mathrm{a}, *}$, Hendrik A. de Weerd ${ }^{\mathrm{b}}$, Katarina Ejeskär ${ }^{\mathrm{a}}$

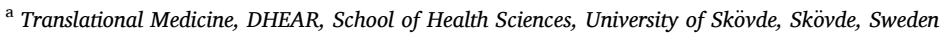 \\ ${ }^{\mathrm{b}}$ School of Bioscience, Systems biology research center, University of Skövde, Skövde, Sweden
}

\section{A R T I C L E I N F O}

\section{Keywords:}

DLG2

DNA Damage

Neuroblastoma

Cancer

\begin{abstract}
A B S T R A C T
Background: In primary neuroblastoma, deletions on chromosome $11 \mathrm{q}$ are known to result in an increase in the total number of chromosomal breaks. The DNA double-strand break repair pathways mediated by NHEJ are often upregulated in cancer. DLG2, a candidate tumor suppressor gene on chromosome 11q, has previously been implicated in DNA repair.

Methods: We evaluated an association between gene expression and neuroblastoma patient outcome, risk categorization, and $11 \mathrm{q}$ status using publicly available microarray data from independent neuroblastoma patient datasets. Functional studies were conducted using comet assay and H2AX phosphorylation in neuroblastoma cell lines and in the fruit fly with UVC-induced DNA breaks.

Results: We show that the NHEJ genes PARP1 and FEN1 are over expressed in neuroblastoma and restoration of $D L G 2$ impairs their gene and protein expression. When exposed to UVC radiation, cells with DLG2 over expression show less DNA fragmentation and induce apoptosis in a p53 S46 dependent manner. We could also confirm that DLG2 over expression results in CHK1 phosphorylation consistent with previous reports of G2/M maintenance.

Conclusions: Taken together, we show that DLG2 over expression increases p53 mediated apoptosis in response to etoposide and UVC mediated genotoxicity and reduced DNA replication machinery.
\end{abstract}

\section{Introduction - DNA repair in neuroblastoma}

Neuroblastoma (NB) is a tumor arising from the embryonic neural crest, later presenting in the autonomous nervous system [1]. It is one of the most common forms of pediatric malignancies and has a disproportionately high mortality rate [2]. Clinical screening and eventual diagnosis of NB is difficult due to the vague appearance of symptoms which is compounded by the young age of the patients [3]. NB can be defined by risk group, both low and intermediate NB have good treatment prospects, whereas high risk tumors are difficult to treat with current protocols [4]. This difficulty to treat results in higher incidence of refractory NB [5] as well as higher mortality [6], indicating that new treatments are needed. High-risk NBs are highly aggressive, with specific genetic lesions, copy number variations (CNV), and structural chromosomal changes [7]. Common genetic features of NB include chromosome $1 \mathrm{p}$ deletion [8-10] and $17 \mathrm{q}$ gain [11,12], whereas $11 \mathrm{q}$ deletion $[7,13,14]$ and amplification of the proto-oncogene MYCN [15-19] account for approximately $30 \%$ and $20 \%$ of all NB cases, respectively. In $\mathrm{NB}$, deletion of $11 \mathrm{q}$ has previously been shown to result in an increase in the number of chromosomes with DNA breaks as well as the total number of chromosomal breaks [7].

DNA strand breaks can be repaired using different mechanisms depending on the type of break. DNA repair of single-strand breaks will often be highly successful as the DNA template still remains. In contrast, double-strand break repair can be deleterious as there is no template remaining for use during DNA repair. There are two main mechanisms by which double-strand break repair occurs: (1) Homologous Repair (HR) is highly reliable and uses the sister chromatid as a template, but can only take place during the S and G2 cell cycle phases [20] and (2) non-homologous end joining (NHEJ) does not use a template and directly ligates the two ends of the DNA breaks, meaning that the repair mechanism can be active throughout the cell cycle, except during

List of abbreviations: MMEJ, microhomology mediated end joining; NB, neuroblastoma; NHEJ, non-homologous end joining; HR, homologous repair; SRO, smallest region of overlap; S46, Serine 46; T68, Threonine 68; CHK, checkpoint kinase.

* Corresponding author.

E-mail address: simon.keane@his.se (S. Keane). 
mitosis [21].

Two distinct mechanisms of NHEJ have been found to occur, namely canonical NHEJ (c-NHEJ) and alternative NHEJ (alt-NHEJ). C-NHEJ is a more accurate DNA repair system, which includes several genes, i.e. XRCC4, XRCC5, XRCC6, DCLRE1C, LIG4 and DNA-PKcs [21-23]. a-NHEJ, also referred to as microhomology-mediated end joining (MMEJ), is highly erroneous as the homology sequences create overhangs which are subsequently removed, creating insertions, deletions and whole chromosomal losses [24,25]. As MMEJ-mediated repair is rare in G1 but increases in S and G2 phases, it is active at the same time as HR. Although cells undergo MMEJ when they cannot successfully undergo HR [26], the repair processes has been shown to be competitive. Genes involved in the MMEJ pathway include MRE11a (11q21), FEN1 (11q12), LIG3 (17q12), XRCC1 (19q13), NBS1 (8q21), and PARP1 (1p42) [24].

\subsection{DNA repair genes are altered in $11 q$ deleted neuroblastoma}

In $\mathrm{NB}$, the 11q-deleted region spans a number of candidate tumor suppressor genes such as ATM, H2AX, CHK1 and MRE11a [6], all of which are involved in DNA damage response (DDR) [27]. Recently, 11q deleted NB was shown to have abnormally low expression of Discs Large Homologue 2 (DLG2; 11q14) [27,28]. Low DLG2 expression has also been found in osteosarcoma [29] and ovarian cancer [30]. Previous results show that $D L G 2$ can regulate the cell cycle by altering cyclin A and B as well as potentially regulating DNA damage repair [27]. All of the candidate tumor suppressors spanning the $11 \mathrm{q}$ region have been shown to lack the second hit as per the Knudson two-hit hypothesis [6]. It has, therefore, previously been suggested that there is a coalescence of haploinsufficiency within a single pathway [28]. However, this has not yet been proven.

In this study, we investigate the difference in expression of genes associated with NHEJ in primary NB of various stages, and can show that some of these changes may be induced by loss of DLG2. Furthermore, we can show that DLG2 loss increases the presence of DNA double-strand breaks, and that restoration of DLG2 results in an increase in overall genomic stability.

\section{Methods}

\subsection{Gene expression analysis}

To compare gene expression between different NB patient subgroups (risk, patient survival, and chromosome 11q status), microarray data (centered $\log 2$ fold change) for primary NB datasets (i.e. Risk group GSE49710 [31], Patient survival GSE16476 [32], 11q GSE3960 [33], and 11q GSE73517 [34]) were downloaded from the R2 platform (http://r2.amc.nl) as $\log 2$ centered values.

\subsection{Cell lines and tissue culture}

Human NB cell lines (SKNAS, SKNBE(2), and NB69) were obtained from the ATCC Cell Line Collection (ATCC). SHSY5y NHEJ reporter cells (DR5000-HNSH-Sce) were obtained from Topogen. SKNAS, SKNBE(2), SHSY-5y were maintained in RPMI 1640 culture medium supplemented with $10 \%$ FBS, $1 \%$ L-Glutamine, 1\% HEPES solution, and $1 \%$ sodium pyruvate. NB69 was cultured in RPMI 1640 medium supplemented with $15 \%$ FBS, $1 \%$ L-Glutamine, 1\% HEPES solution, and 1\% sodium pyruvate. The cells were cultured in a humidified incubator at $37^{\circ} \mathrm{C}$ with $5 \%$ $\mathrm{CO}_{2}$.

\subsection{Plasmids, siRNAs, transfections, irradiation and treatment}

The DLG2 isoform 7 (NM_001351274.2) over expression plasmid on a backbone of pcDNA3.1/C-(K)-DYK (OHuq102626D) vector was purchased from GenScript (Genscript Biotech Corporation). siRNA targeting
Table 1

List of genes and probe ID used for qPCR Investigation.

\begin{tabular}{ll}
\hline Gene name & Probe ID \\
\hline DLG2 & Hs00265843_m1 \\
BAX & Hs00180269 \\
BCL2 & Hs00608023 \\
PARP1 & Hs002422302 \\
MRE11a & Hs00967437 \\
FEN1 & Hs00748727 \\
XRCC1 & Hs00959834 \\
LIG3 & Hs00242692 \\
NBS1 & Hs00159537 \\
GAPDH & Hs02758991 \\
GUSB & Hs99999904 \\
dmDLG & Dm01799281_g1 \\
PARP & Dm03419822 \\
MRE11 & Dm01817703 \\
FEN1 & Dm01821494 \\
XRCC1 & Dm795840 \\
LIG3 & DM02139557 \\
Rpl32 & Dm02151827 \\
\hline
\end{tabular}

DLG2 (cat. s4122) or Silencer ${ }^{\mathrm{TM}}$ Select Negative control No. 1 siRNA (cat. 4390843) were purchased from Ambion (Thermo Fisher Scientific). Cell lines were grown to $80 \%$ confluence and subsequently transfected with DLG2 plasmid, empty vector "mock" (pCMV6-AC-GFP or pCMV6Myc-DDK), si-DLG2 or scrambled control "mock". In brief, $500 \mathrm{ng}$ plasmid DNA or 30 pmol siRNA was complexed with $12 \mu$ l Lipofectamine 2000 according to the manufacturer's protocol (Thermo Fisher Scientific). To induce DNA damage, the cells were uncovered and subjected to UVC radiation (ESCO; CRF/UV-30A; $253.7 \mathrm{~nm}$ ) for $30 \mathrm{~s}$ at $1200 \mathrm{~mm}$, immediately given fresh media, and allowed to recover for $2 \mathrm{~h}$ or $24 \mathrm{~h}$ prior to harvesting and compared to control cells. As a second method to induce DNA damage, cells were treated with $7.5 \mu \mathrm{M}$ etoposide (Merck) for $24 \mathrm{~h}$ and subsequently harvested.

\subsection{Comet assay}

To determine DNA damage repair post UV exposure or etoposide treatment, comet assay was performed according to the manufacturer's protocol (Abcam, Cat. ab238544). Aliquots of 10000 cells were mixed with low melting point agarose and added to comet assay microscope slides. The slides were allowed to harden for $15 \mathrm{~min}$, lysed $\left(\mathrm{pH} 10 ; 4^{\circ} \mathrm{C}\right)$ for $1 \mathrm{~h}$ in pre-chilled lysis buffer, subsequently washed 3 times in alkaline buffer and transferred into alkaline buffer for electrophoresis. The slides were run for $30 \mathrm{~min}$ at $1 \mathrm{~V} / \mathrm{cm}, 300 \mathrm{~mA}$, washed 3 times with $\mathrm{H}_{2} \mathrm{O}$, incubated for 5 min with ice cold $70 \%$ ethanol, allowed to air dry, and incubated with Vista green for visualization. Measurements and analysis were performed using Image $J$ software version 1.52a. Data were normalized to the control transfection and expressed as a percentage of the control.

\subsection{Quantitative PCR ( $q P C R)$ analysis}

Total RNA from NB cell lines and flies was extracted using the RNeasy plus mini kit ${ }^{\circledR}$ (Qiagen) according to the manufacturer's protocol. The RNA concentration was quantified by NanoDrop (NanoDrop Technologies) and $2 \mu \mathrm{g}$ of RNA was reverse transcribed into double stranded cDNA on a T-professional Basic Gradient thermal cycler (Biometra) using the High Capacity cDNA Reverse Transcription kit (Applied Biosystems). cDNA corresponding to $20 \mathrm{ng}$ of RNA for each qPCR reaction was used. qPCR was performed on a Pikoreal qPCR System (Thermo Fisher Scientific) in triplicate for TaqMan Human and Drosophila transcripts (Table 1), using TaqMan ${ }^{\mathrm{TM}}$ Gene Expression Master Mix (Applied Biosystems, Cat. 4318157). Quantitative gene expression data were normalized to the expression levels of the two human reference genes GAPDH and GUSB, or the fly reference gene Rpl32 (Table 1) using the Livak method $[35,36]$. 

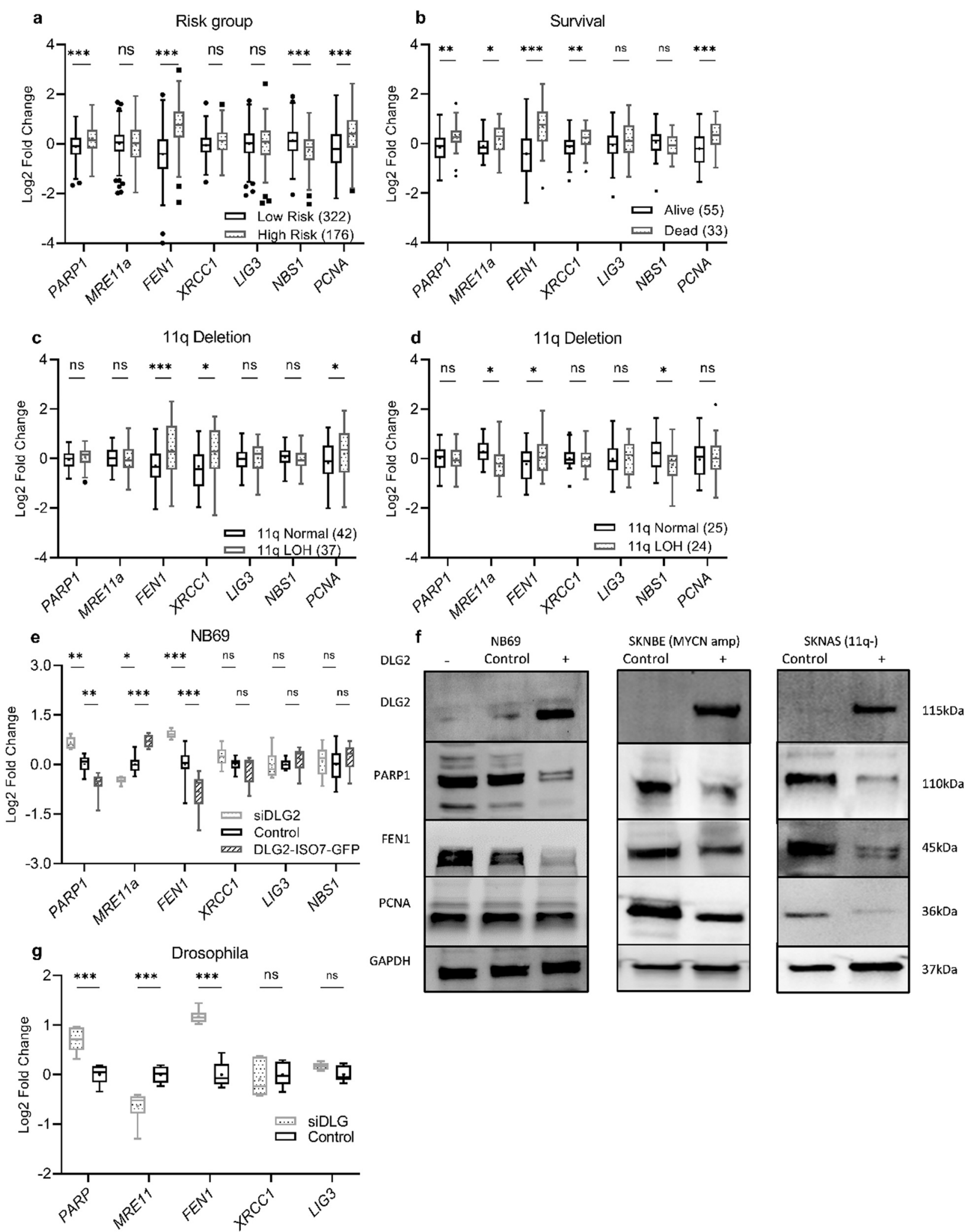

Fig. 1. NB patients show differential NHEJ gene expression based on clinical classifiers. Microarray-based gene expression profiling of NHEJ genes in primary NB samples stratified by a Risk group (GSE49710), b survival (GSE16476), and 11q status c (GSE3960) and d (GSE73517). e qPCR 48 h post DLG2 over expression (DLG2 -DYK) or silencing (siDLG2) in NB69 cells $(\mathrm{n}=9)$ with GAPDH and GUSB as endogenous controls, pooled average of three experiments. $\mathrm{f}$ Immunoblot in NB69 (MYCN and 11q normal), SKNBE (MYCN amplification) and SKNAS (11q loss) cells for DLG2, PARP1, FEN1, PCNA and GAPDH; - = DLG2 silenced, Mock = control, and $+=D L G 2$ over expressed. $\mathrm{g}$ qPCR quantification of Drosophila NHEJ genes in fruit flies with silenced dmDLG expression (siDLG) (n = 9) with RPL32 as an internal control. The expression data are presented as median centered log2 fold change and plotted as Tukeys box and whisker plots showing IQR, line at the median, + at the mean with whiskers \pm 1 .5-fold of interquartile range. Data outside the whiskers are shown as outliers. Statistical significance was determined by one-way ANOVA with Tukey's multiple comparison test. $* P<0.05,{ }^{* *} P<0.01,{ }^{*} * P<0.001$, ns $=$ not significant. 


\subsection{Apoptosis assay}

Apoptosis levels were determined in the transfected NB cells to confirm the previous qPCR results by the Apo-ONE® Homogeneous Caspase-3/7 Assay (Promega) by exciting at $485 \mathrm{~nm}$ and reading at 520 nm using a FLUOstar Omega multiplate reader (BMG Labtech), as per the manufacturer's instructions. After the addition of the caspase assay, the plate was incubated for $65 \mathrm{~min}$ at room temperature. Apoptosis was calculated by subtracting the fluorescence for the average blank background and then normalizing against the mean of the control cells.

\subsection{NHEJ reporter cells}

The relative level of NHEJ was determined using SHSY5y NHEJ reporter cells (DR5000-HNSH-Sce). The cells were transfected as previously described and the number of GFP positive cells quantified $48 \mathrm{~h}$ post transfection by microscopy at 20x magnification on the EVOS M7000 (Thermofisher). The number of positive cells was then normalized to the control and expressed as log2 Fold change.

\subsection{Fly strains and crosses}

Commercially available control white (w1118) flies (Drosophila melanogaster) and UAS-RNAi-dlg1 flies were crossed with da-GAL4 driver strain to silence gene expression. All strains were obtained from the Bloomington Drosophila Stock Center (Bloomington). Twenty female da-GAL4 flies were crossed with 10 male UAS-transgenic flies or control flies and the progeny incubated at $25^{\circ} \mathrm{C}$ on standard fly media. 10 flies per cross were subsequently harvested for subsequent RNA and DNA preparation. To determine the effect of UVC irradiation the flies were subject to 30 Seconds UVC and $3 \mathrm{~h}$ recovery on regular substrate.

\subsection{Nanopore libraries and Sequencing}

To prepare ONT libraries $D$. melanogaster gDNA was extracted with the Blood \& Cell Culture DNA Kit (13323, Qiagen) using the manufacturer's tissue protocol. Libraries were prepared with $1 \mu \mathrm{g}$ gDNA using the Ligation Sequencing Kit SQK-LSK109 (Oxford Nanopore Technologies) combined with native barcoding expansion 1-12 PCR free EXPNBD104 (Oxford Nanopore Technologies) according to the manufacturer's protocol. Two $24 \mathrm{~h}$ sequencing runs for D. melanogaster were performed with R9.4.1MinION flow cells.

\subsection{Sequence alignment and quantification of DNA integrity}

To quantify DNA breakage, a strategy of global alignment between the full length of the sequenced reads and the corresponding reference genome region, where the read best aligned was chosen. Initial alignment was performed with BLASTn version 2.9.0+. A custom BLAST reference database was created from FlyBase $D$. melonagaster version 6.38 (FB2021_02, released April 13, 2021) [37], filtered to only contain chromosomes. All reads passing quality control were subsequently BLASTed to this database using a gap opening and extension penalty of 1 and an e value cutoff of $1 \mathrm{e}-10$. Only hits that uniquely mapped to the reference genome were considered for further downstream alignment. Global alignment was performed using the needle function from the Biopython module EMBOSS [38] on the full length of the read and the corresponding region of the same length in the reference genome. Every mismatch, insertion and deletion were counted in these alignments. To quantify fused sequences, previously discarded reads were counted that fit the following criteria: the read has exactly two BLAST hits and there is at least $10 \mathrm{~kb}$ between the two hits to account for larger deletions in the sequenced DNA.

\subsection{Western blot}

Total protein was extracted from transfected cells in 24 well plates $\left(1 \times 10^{5}\right.$ cells/well), by aspirating the media and incubating on ice for 5 min then adding ice cold RIPA buffer (Thermo Fisher Scientific, Cat. 89900). Western blot analysis was performed using a Mini-PROTEAN $®$ $\mathrm{TGX}^{\mathrm{TM}} 8-20 \%$ gradient gel (Bio-Rad Laboratories), and protein blotted onto LF-PVDF membrane ( $8 \mathrm{~min}, 25 \mathrm{~V}$ and $2.5 \mathrm{~A}$ ) using a Trans-Blot ${ }^{\circledR}$ Turbo $^{\mathrm{TM}}$ Transfer System (Bio-Rad Laboratories). Blots were subsequently blocked for $1 \mathrm{~h}$ in $5 \%$ milk in Tris buffered saline (TBS) $(150 \mathrm{mM}$ $\mathrm{NaCl}$ in $10 \mathrm{mM}$ Tris-HCL, pH 7.4) buffer supplemented with $0.1 \%$ Tween-20, as per the manufacturer's recommendations. Blots were probed overnight at $4{ }^{\circ} \mathrm{C}$ with antibodies diluted in PBST $(0.1 \%$ Tween20 in phosphate buffered solution (PBS)). Primary antibodies for DLG2 (1:500, Cell Signaling Technology, Cat. D4Z4D), PARP1 $(0.5 \mu \mathrm{g} / \mathrm{ml}$, DSHB Hybridoma, Cat. AFFN-PARP1-17B10 was deposited to the DSHB by EU Program Affinomics), FEN1 (1:1000, SCBT, Cat. sc-28355), PCNA (1:1000, SCBT, Cat. sc-56), H2AX (1:1000, SCBT, Cat. sc-517336), $\gamma \mathrm{H} 2 \mathrm{AX}$ (1:1000, SCBT, Cat. sc-517348), P53 (1:1000, SCBT, Cat. sc126), S46 P53 (1:1000, SCBT, Cat. sc-377561), BAX (1:1000, SCBT, Cat. sc-20067), BCL2 (1:1000, SCBT, Cat. sc-509), CHK1 (1:1000, SCBT, Cat. sc-8408), S317 CHK1 (1:1000, CST, Cat. D12H3), CHK2 (1:1000, SCBT, Cat. sc-5278), T68 CHK2 (1:1000, CST, Cat. C13C1), ATM (1:1000, SCBT, Cat. sc-135663), DNA-PKcs (1:1000, CST, Cat. E6U3A) and GAPDH (1:2500, Bio-Rad, Cat. 12004168). The secondary antibodies used for detection were Starbright B520 goat anti-Rabbit (1:5000, Bio-Rad, Cat. 12005870), Starbright B700 goat anti-mouse (1:5000, Bio-Rad, Cat. 12004159), both of which were diluted in PBST $0.1 \%$. All wash stages were $3 \times 10 \mathrm{~min}$ in TBST $0.1 \%$. Secondary antibodies were incubated for $1 \mathrm{~h}$ at room temperature. Image detection was performed on ChemiDoc MP (Bio-Rad Laboratories) with image analysis performed using Image Lab (Version 6.0.0 build 25, Bio-Rad Laboratories).

\subsection{Statistical analysis}

All data are presented as Tukey's box and whisker plots showing IQR, line at the median, + at the mean with whiskers \pm 1.5 -fold of interquartile range for at least 3 independent experiments. For all multigroup analyses, differences were determined by one-way ANOVA test followed by Holm-Sidak's multiple comparison test. For comparisons between two groups, Mann-Whitney U test was performed. A p $<0.05$ was considered to be statistically significant. All analyses were conducted using GraphPad Prism version 9.0.1 for Windows (GraphPad Software, www.graphpad.com).

\section{Results}

3.1. NHEJ gene expression was dysregulated in high risk NB, and could be reversed by DLG2 over expression

We evaluated the expression of NHEJ genes using publicly available microarray data for four independent NB patient datasets (GSE49710, GSE16476, GSE3960, and GSE73517) obtained from the R2 Genomics Analysis and Visualization Platform (http://r2.amc.nl), and stratified according to risk status (Fig. 1a), patient survival (Fig. 1b), or 11q deletion status (Fig. 1c, d). High-risk NB showed elevated expression of PARP1 $(\log 2 \mathrm{FC}=0.337, p<0.001)$, FEN1 $(\log 2 \mathrm{FC}=1.17, p<0.001)$, and PCNA $(\log 2 \mathrm{FC}=0.595, p<0.001)$ with lower NBS1 expression in high risk tumors $(\log 2 \mathrm{FC}=-0.368, p<0.001)$ (Fig. 1a). The expression of these genes was also associated with poor survival, as elevated PARP1 $(\log 2 \mathrm{FC}=0.454, p=0.005), F E N 1(\log 2 \mathrm{FC}=1.09, p<0.001)$, XRCC1 $(\log 2 \mathrm{FC}=0.352, p=0.04)$, and PCNA $(\log 2 \mathrm{FC}=0.544, p<0.001)$ expression were associated with higher incidence of mortality (Fig. 1b). Overall survival was also determined by Kaplan-Meier for each of the previously discussed genes (Appendix A) with PARP1, FEN1 and PCNA 
a

Head

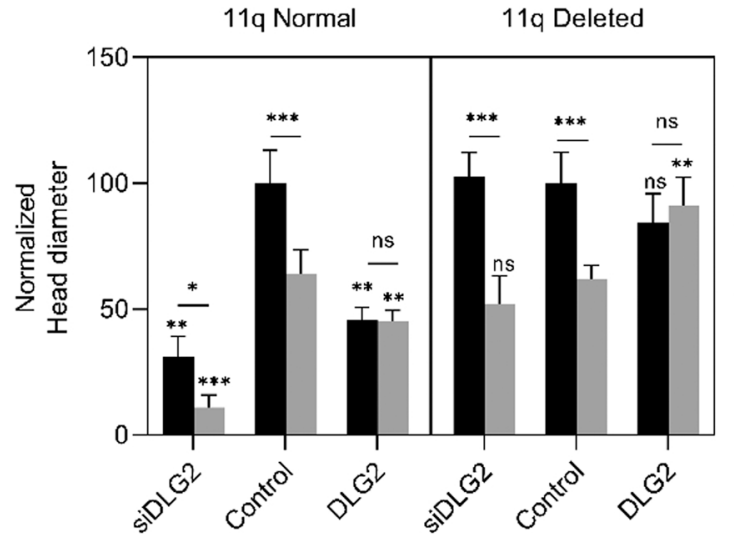

c NHEJ activation

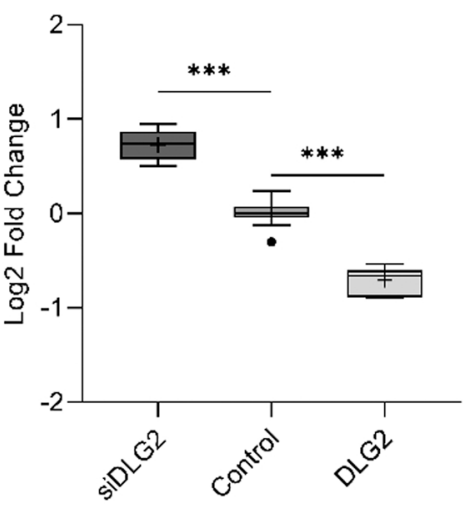

e Fusion Sequences

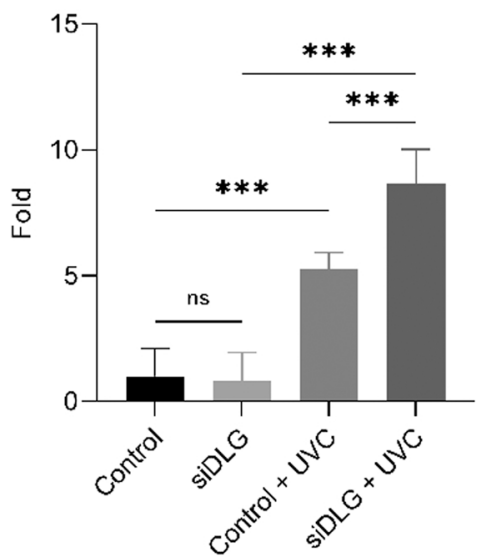

b
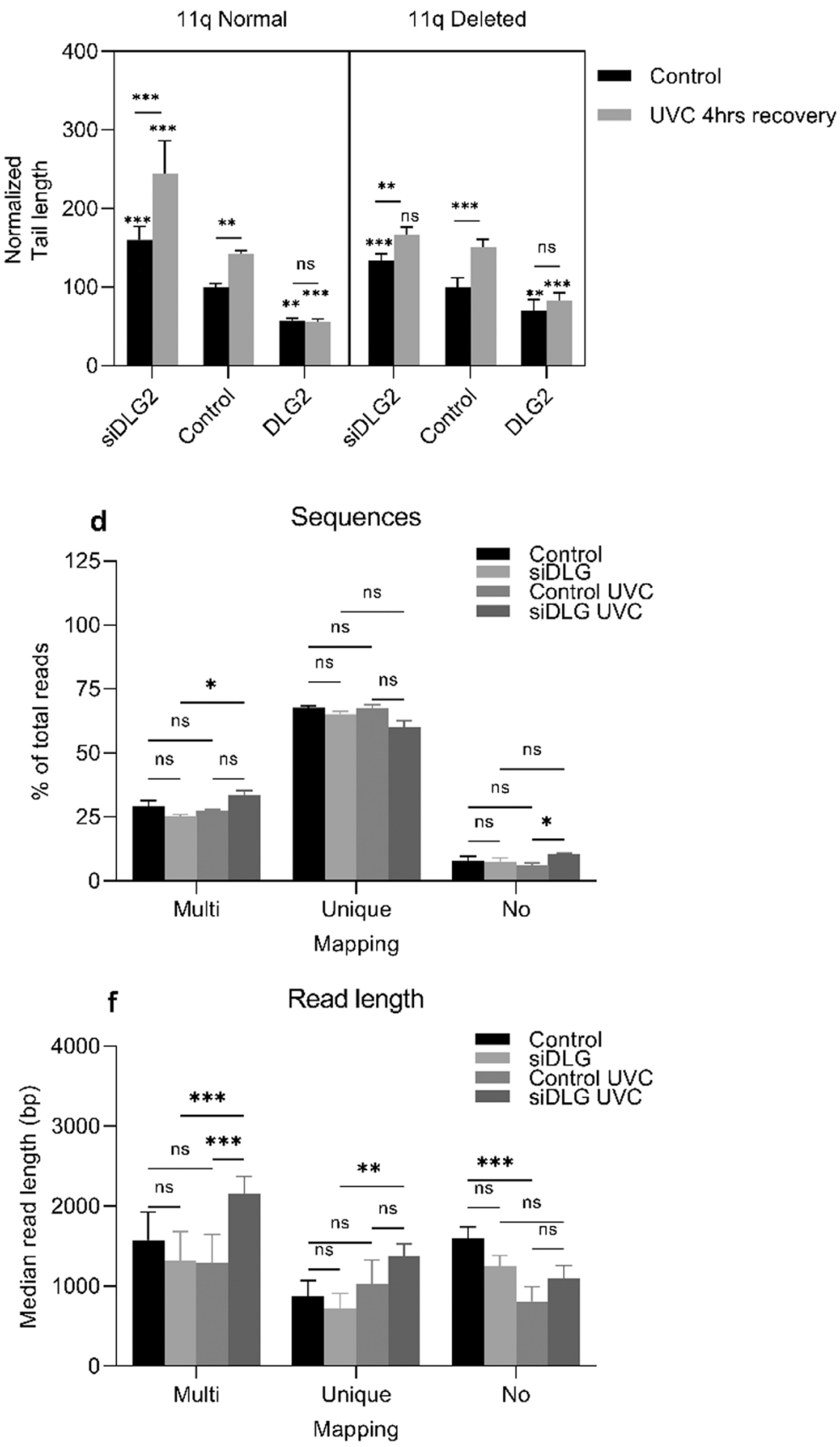

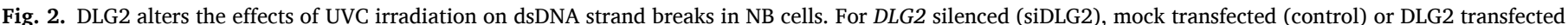

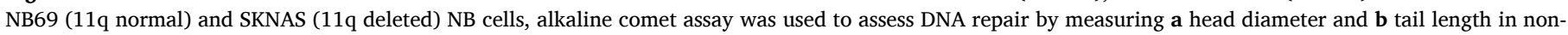

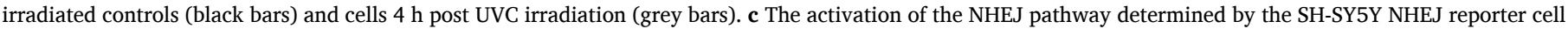

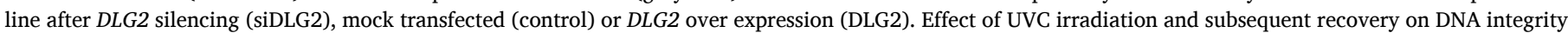

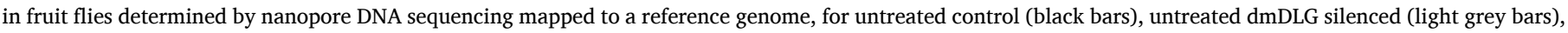

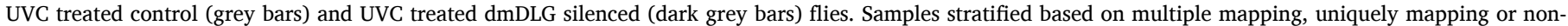

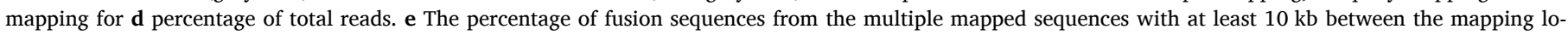

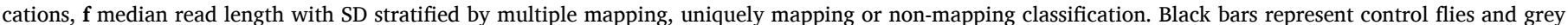

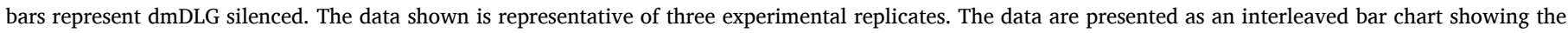
mean and SD. Significance was determined by two-way ANOVA with Šídák's multiple comparison test ${ }^{*} P<0.05$, $* * P<0.01$, $* * * P<0.001$, ns $=$ not significant.

showing lower expression resulting in better survival. In tumors harboring 11q deletion, FEN1 ( $\log 2 \mathrm{FC}=0.636, p<0.001$ ), XRCC1 $(\log 2 \mathrm{FC}=0.549, p<0.05)$, and PCNA $(\log 2 \mathrm{FC}=0.378, p<0.01)$ were up regulated compared to $11 \mathrm{q}$-normal tumors in the GSE3960 dataset
(Fig. 1c). MRE11a and NSB1 were significantly down regulated in the GSE73517 dataset $(\log 2 \mathrm{FC}=-1.335, p=0.013$ and $\log 2 \mathrm{FC}=-1.335$, $p=0.013$; Fig. 1d). FEN1 ( $\log 2 \mathrm{FC}=0.427, p<0.05)$ was over expressed in the $11 \mathrm{q}$ deleted tumors in both $11 \mathrm{q}$ deleted datasets (Fig. 1c-d). 
a BAX
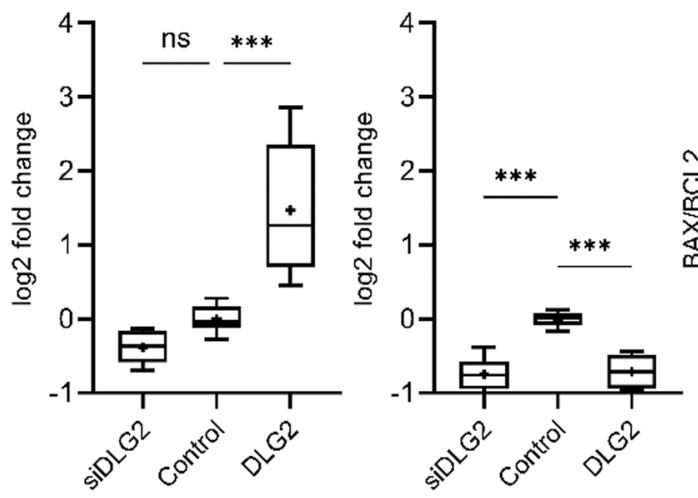

c $\mathrm{BAX} / \mathrm{BCL} 2$

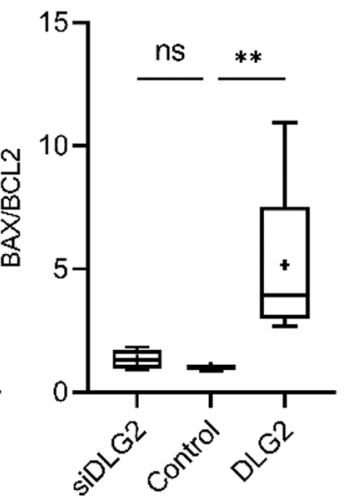

d
Caspase $3 / 7$ activation
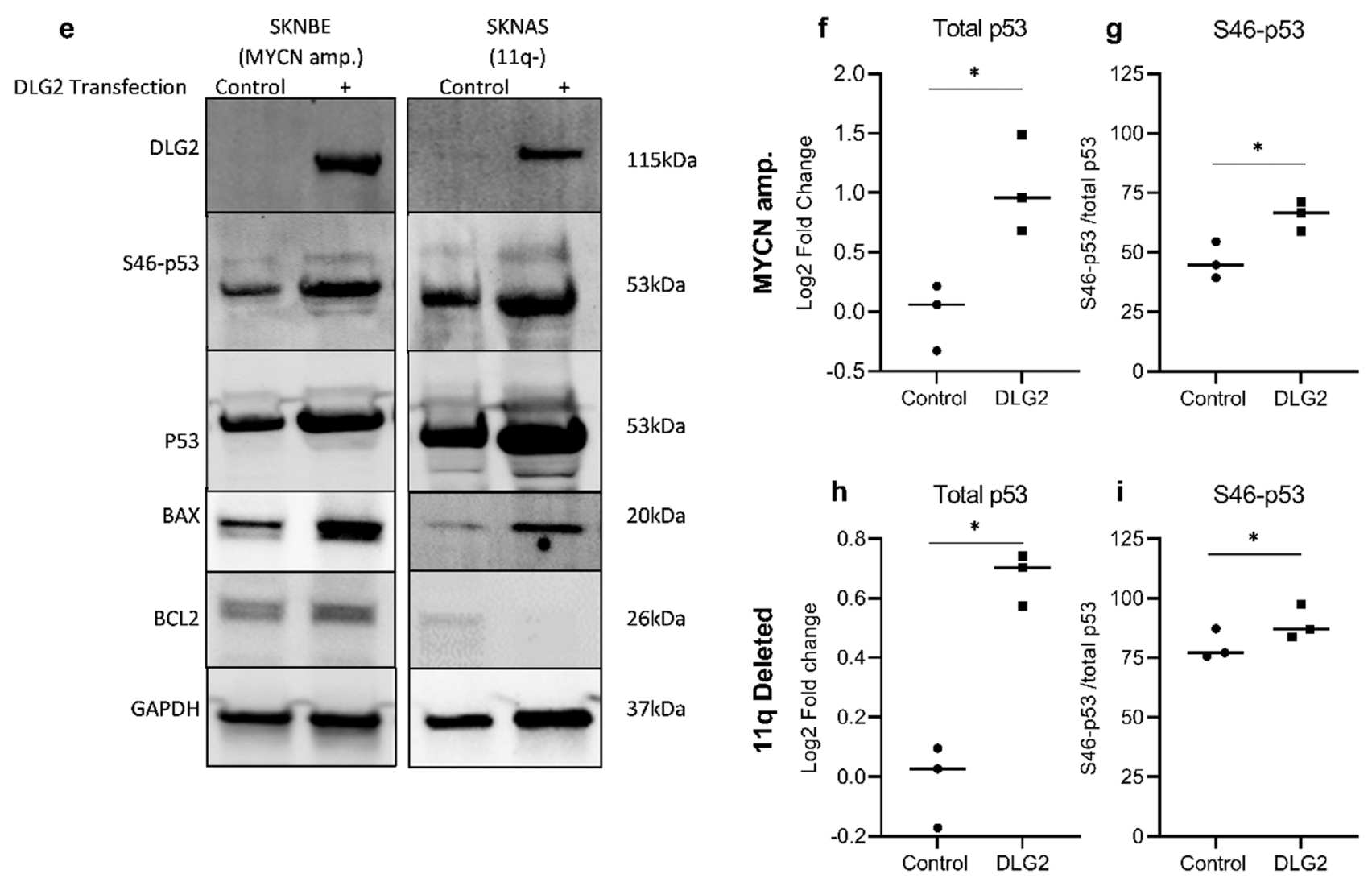

$53 \mathrm{kDa}$

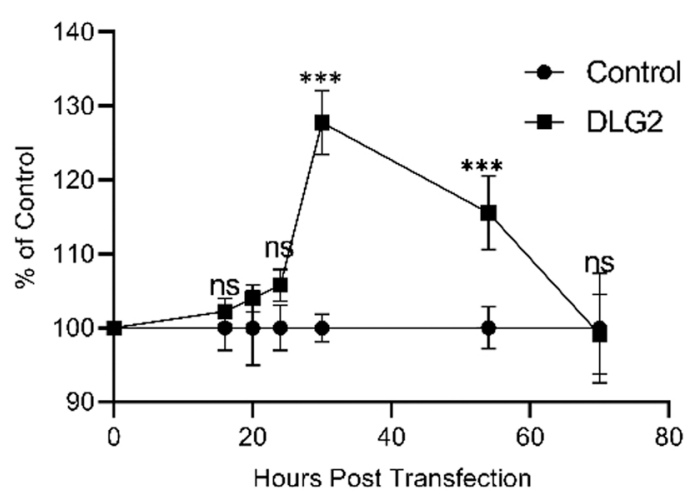

Fig. 3. DLG2 induces apoptosis in NB in a p53 dependent manner. a BAX, b BCL2, and $\mathbf{c} B A X / B C L 2$ gene expression analysis by qPCR $48 \mathrm{~h}$ post $D L G 2$ over expression (DLG2) or silencing (siDLG2) in 11q deleted NB SKNAS cells. The log2 fold change was determined using the Livak method normalized to the mean of GAPDH and GUSB. d Apoptosis induction by DLG2 over expression compared to control measured by Caspase 3/7-level over time in SKNAS cells. e DLG2, BAX, BCL2, p53, and pSer46 p53 protein expression measured by immunoblot in SKNBE (MYCN amp.) and SKNAS (11q Deleted) cells; $+=$ DLG2 over expression. Total p53 expressed as $\log 2$ Fold Change and S46 p53/ Total p53 protein levels quantified by optical density in SKNBE (f-g) and SKNAS (h-i) cells. All data is the pooled average of three experiments. The data in $\mathbf{c}$ and $\mathbf{d}$ are shown as the mean \pm SD. The data in a-c are presented as Tukeys box and whisker plots showing IQR, line at the median, + at the mean with whiskers \pm 1.5 -fold of interquartile range. Significance was determined by (a-c) one-way ANOVA with Dunnett's multiple comparison test and (f-i) unpaired two-tailed Student's t-test. ${ }^{*} P<0.05,{ }^{*} * P<0.01,{ }^{*} * * P<0.001, \mathrm{~ns}=$ not significant.

In the NB69 NB cell line (11q normal), we could show that over expression of DLG2 resulted in down regulation of PARP1 and FEN1 $(\log 2 \mathrm{FC}=0.594, p=0.001$ and $\log 2 \mathrm{FC}=0.818, p=0.001)$ and up regulation of MRE11a $(\log 2 \mathrm{FC}=0.704, p<0.001)$, whereas DLG2 knockdown resulted in increased PARP1 $(\log 2 \mathrm{FC}=0.637, p=0.001)$, FEN1 $(\log 2 \mathrm{FC}=0.925, p=0.001)$ and decreased MRE11 $a$ expression ( $\log 2 \mathrm{FC}=0.474, p=0.01$ ) (Fig. 1e). We verified that the changes in PARP1, FEN1, and PCNA gene expression in NB69 (MYCN and 11q normal) correlated to the protein expression levels, after DLG2 over expression and silencing, as well the effect of $D L G 2$ over expression on these genes in the NB cell lines SKNBE (MYCN amplified) and SKNAS (11q deleted) (Fig. 1f). To further evaluate these findings, we performed a general knockdown of $d m D L G$ using RNAi in Drosophila melanogaster where we could confirm that loss of $d m D L G$ resulted in increased PARP $(\log 2 \mathrm{FC}=0.702, p<0.001)$ and FEN1 $(\log 2 \mathrm{FC}=1.170, p<0.001)$ expression, as well as down regulation of MRE11a $(\log 2 \mathrm{FC}=0.634$, $p<0.001$ ) (Fig. 1g). Expression of NHEJ genes XRCC1 and LIG3 were not affected by $D L G 2$ over expression or silencing in NB cells or the fly 

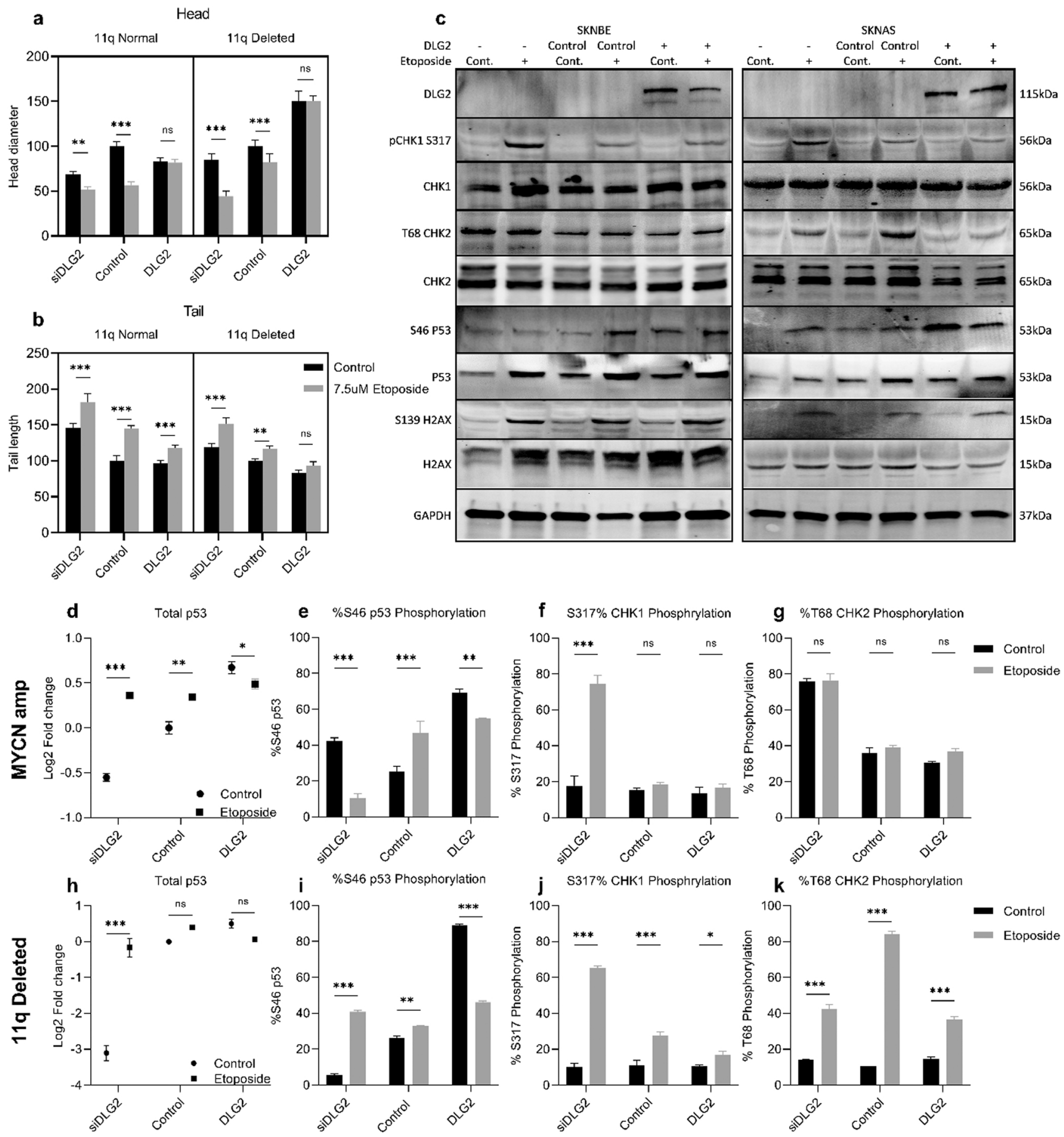

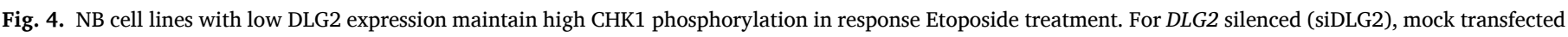

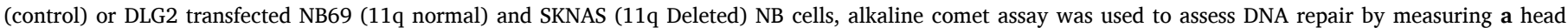

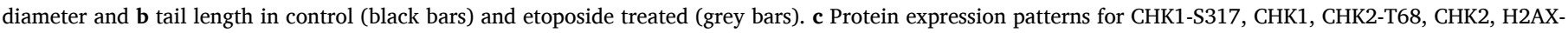

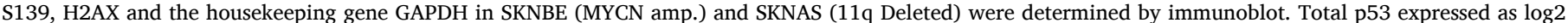

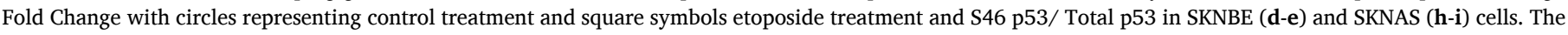

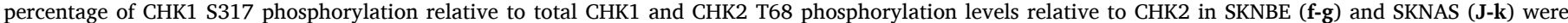

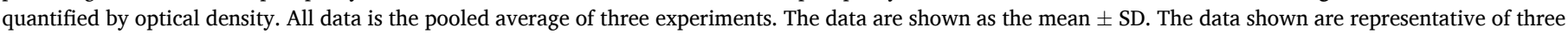
experimental replicates. ${ }^{*} P<0.05$, ${ }^{*} P P<0.01$, ${ }^{*} * * P<0.001$, ns $=$ not significant.

model (Fig. 1e, g).

\subsection{DLG2 enhanced DNA repair in NB cells after UVC irradiation}

To determine whether NB cells with or without 11q-deletion had more tendency to accumulate DNA breaks, we induced DNA breaks by exposure to UVC in combination with a forced change in DLG2 expression level. DNA integrity was assessed by exposing DLG2 transfected NB cells to UVC irradiation followed by comet assay. We could determine that silencing of DLG2 resulted in a smaller comet assay head size $(68.7 \%, p<0.001)$ and longer tail length $(60.1 \%, p<0.001)$ in $11 \mathrm{q}$ normal NB cells prior to UVC irradiation. Silencing DLG2 in 11q deleted 
cells resulted in no change in head size $(15.0 \%, \mathrm{p}=0.21)$ but an increased tail size $(33.3 \%, p<0.001)$, indicating fewer intact DNA strands and more DNA breaks in cells with lower DLG2 levels (Fig. 2a, b). A decrease in head size $(54.1 \%, p<0.001)$ was also detected in the DLG2 over expressed cells before UVC irradiation in 11q normal NB cells. However, tail length was shown to be decreased in both $11 \mathrm{q}$ normal $(43.2 \%, p<0.001)$ and $11 \mathrm{q}$-deleted $(29.4 \%, p<0.001)$ cells with $D L G 2$ over expression prior to UVC irradiation, indicating a general effect on the amount of DNA breaks without inducing breaks (Fig. 2b). To determine the effect of DLG2 on DNA repair, we induced DNA breaks with UVC irradiation in 11q-deleted and 11q-normal NB cells and allowed the cells to recover for $4 \mathrm{~h}$. Using comet assay, we could determine that there was a decrease in the head size in both 11q-normal and 11q-deleted cells after irradiation in DLG2 silenced cells $(20.1 \%$, $p<0.001 \%$ and $50.4 \%, p<0.001$ ) and control samples $(36.1 \%$, $p<0.001 \%$ and $38.2 \%, p<0.001$ ); no difference was seen between the irradiated and non-irradiated samples when DLG2 was over expressed (Fig. 2a, b). There was a corresponding increase in the length of the tail in these samples; DLG2 silenced cells $(83.8 \%, p<0.001$ and $33.9 .4 \%$, $p<0.01)$ and control samples $(42.7 \%, p<0.001 \%$ and $51.6 \%$, $p<0.001$ ), with no increase in the tail length of the DLG2 over expressed cells (Fig. 2b). To verify our hypothesis that it was the level of non-homologous end joining (NHEJ) that was affected by DLG2 expression, we used the SH-SY5y NHEJ reporter cell line with over expressed $D L G 2$, mock or $D L G 2$ silencing. Silenced $D L G 2$ resulted in an increase in NHEJ active cells compared to control $(\log 2 \mathrm{FC}=0.727$, $p<0.001$ ) with the over expression of DLG2 resulting in a decrease in the activation of NHEJ $(\log 2 \mathrm{FC}=-0.706, p<0.001$; Fig. $2 \mathrm{c})$.

To further determine the effect of UVC irradiation on DNA breakage and $D L G$ loss we compared the DNA integrity after UVC irradiation in a Drosophila model with RNAi-silenced DLG (dmDLG knockout) to controls. We evaluated the percentage of DNA-sequences that were multiple, unique and not mapped (Fig. 2d) in UVC treated and untreated control (white) and $d m D L G$ knockout flies. We could ascertain that there were more unmapped sequences in the UVC treated, $d m D L G$ knockout flies compared to the untreated $d m D L G$ knockout $(7.8 \%$ increase over the control, $\mathrm{p}<0.05)$. There was no difference in the percentage of mapped sequences for any of the samples. DNA from the UVC treated $d m D L G$ knockout samples had a higher percentage of non-mapped sequences compared to the UVC treated control (4.33\% increase over the control, $\mathrm{p}<0.01$; Fig. 2d). We further we determined the number of multiple mapped sequence reads that contained two separate fragments from non-overlapping (greater then 10,000 bp gap) sequences. We could show that the untreated control and untreated $d m D L G$ knockout resulted in no difference in the percentage of detected fusion sequences $(0.18$ fold, $p=0.61$ ), there was a large increase in the number of fusion sequences when the control and $d m D L G$ knockout UVC treated samples were compared against their untreated control ( 3.4 fold, $\mathrm{p}<0.001$ and 4.3 fold, $\mathrm{p}<0.001$, respectively). $d m D L G$ knockout after UVC showed a large increase in the number of fusion samples when compared to the UVC treated control (7.9 fold increase, $\mathrm{p}<0.001$; Fig. 2e).

Finally, we determined the median sequence length for the multiple, unique and not mapped sequences for each of the four experiments. The UVC $d m D L G$ knockout flies showed a longer multiple mapped sequences read length compared to the untreated $d m D L G$ knockout flies and the treated control flies ( $836 \mathrm{bp}$ longer, $\mathrm{p}<0.001$ and $868 \mathrm{bp}$ longer, $\mathrm{p}<0.001$, respectively; Fig. 2f) with no significant differences between the other samples. The median read length for UVC $d m D L G$ knockout flies was longer compared to the untreated $d m D L G$ knockout flies ( 654 bp longer, $\mathrm{p}<0.01$ ) with no differences between the other samples. The median length for the unmapped sequences was longer for the untreated control compared against the UVC treated control (788 bp longer, $\mathrm{p}<0.001$ ) with no difference between the other samples.

\subsection{DLG2 over expression induced apoptosis in NB cells}

We evaluated the effect of over expression of DLG2 on the initialization of apoptosis in SKNBE (MYCN-amplified) and SKNAS (11qdeleted) NB cells $48 \mathrm{~h}$ post transfection. Overexpression of DLG2 in SKNAS cells resulted in increased mRNA expression of $B A X(\log 2 \mathrm{FC}=$ $1.47, p<0.001)$ as well as decreased $B C L 2$ expression ( $\log 2 \mathrm{FC}=0.713$, $p<0.001$ ); DLG2 silencing resulted in decreased mRNA expression of $B C L 2(\log 2 \mathrm{FC}=0.744 p=0.001)$ with no alteration in $B A X$ expression (Fig. 3a, b). The ratio of $B A X / B C L 2$ gene expression was determined and shown to be high in cells with DLG2 over expression $(\mathrm{FC}=4.16$, $p<0.01$ ), with no alteration for DLG2 silenced cells $(\mathrm{FC}=0.329$, $p=0.931$; Fig. 3c). To determine if this resulted in apoptosis initialization we determined caspase $3 / 7$ activation over time between 0 and $70 \mathrm{~h}$. There was no difference in caspase $3 / 7$ activation between control cells and DLG2 transfected cells $0,16,20$ or 24 h post transfection; there was an increase in activation at $30 \mathrm{~h}(27.8 \%, p<0.001)$ and $54 \mathrm{~h}$ $(15.6 \%, p<0.001)$, with no difference in the level of activation again at $70 \mathrm{~h}$ (Fig. 3d). We could confirm that $B A X$ gene expression changes after $D L G 2$ over expression resulted in increased levels of BAX protein in both SKNBE and SKNAS cells, and that BCL2 protein levels were lowered in SKNAS cells (Fig. 3e). DLG2 over expression resulted in an increase in total p53 level and increased degree of p53 Ser46 phosphorylation in both SKNBE $(\log 2$ Fold change $=1.0, p<0.05 \%$ and $19.3 \%$ increase compared to the control, $p<0.05$; Fig. $3 \mathrm{f}-\mathrm{g}$ ) and SKNAS (log2 Fold change $=0.69, p<0.01 \%$ and $9.4 \%$ increase compared to the control, $p<0.01$; Fig. 3h-i) cell lines. These results indicated that DLG2 over expression could induce apoptosis in NB cells.

\subsection{DLG2 over expression increases S46-p53 after exposure to etoposide}

To confirm if UVC and etoposide resulted in the same effect on NB cells, we altered DLG2 expression level in cells with or without 11q-deletion to determine if DNA breaks accumulated. DNA integrity was assessed after exposing DLG2 transfected NB cells to $7.5 \mu \mathrm{M}$ etoposide with no recovery period followed by comet assay. We could determine that there was a decrease in the head size in both 11q-normal and $11 \mathrm{q}$ deleted cells after etoposide treatment in DLG2 silenced cells (16.6\%, $p<0.01 \%$ and $41.8 \%, p<0.001$ ) and control samples $(43.3 \%$, $p<0.001 \%$ and $17.6 \%, p<0.001$ ); no difference was seen after etoposide treatment when DLG2 was over expressed (Fig. 4a). There was a corresponding increase in the length of the tail in these samples; DLG2 silenced cells $(35.5 \%, p<0.001 \%$ and $32.1 \%, p<0.001)$ and control samples (45.4\%, $p<0.001 \%$ and $16.6 \%, p<0.01$ ), with no increase in the tail length of the DLG2 over expressed 11q deleted cells, although an increase in the $11 \mathrm{q}$ normal cells $(21.2 \%, p<0.001)$ (Fig. 4b).

To confirm the post translational modifications caused by etoposide treatment we quantified the degree of phosphorylation of CHK1-S317, CHK2-T68, P53-S46, H2AX-S139 and the total protein, with the representative immunoblots from SKNBE (11q normal) and SKNAS (11q deleted) shown in Fig. 4c. Treatment of SKNBE with 7.5 $\mu \mathrm{M}$ etoposide resulted in an increased total p53 expression in control (Log2 Fold change $0.35<0.01$ and siDLG2 Log2 Fold change $0.91<0.001$; Fig. 4d) and a decrease in DLG2 (Log2 Fold change $0.18<0.05$; Fig. 4d) The degree of S46 p53 phosphorylation decreased with etoposide treatment in DLG2 silenced cells $(32.1 \%$ less phosphorylation, $p<0.001)$. The over expression of DLG2 resulted in a high degree of S46 phosphorylation of p53 that was slightly lowered after etoposide treatment $(14.3 \%$ less phosphorylation, $p<0.05$; Fig. $4 \mathrm{e}$ ). The degree of S317- CHK1 phosphorylation was increased by etoposide treatment in DLG2 silenced cells (41.8\% more phosphorylation, $p<0.001$; Fig. 4 f), this was not seen in control or DLG2 over expressed SKNBE cells. The degree of T68-CHK2 phosphorylation was not affected by etoposide in SKNBE cells (Fig. 4g). Treatment of the 11q-deleted SKNAS cells with $7.5 \mu \mathrm{M}$ etoposide resulted in an increased total p53 protein level in DLG2 silenced cells (Log2 Fold change $=2.9, p<0.001$; Fig. $4 \mathrm{~h}$ ). The degree of S46 
a
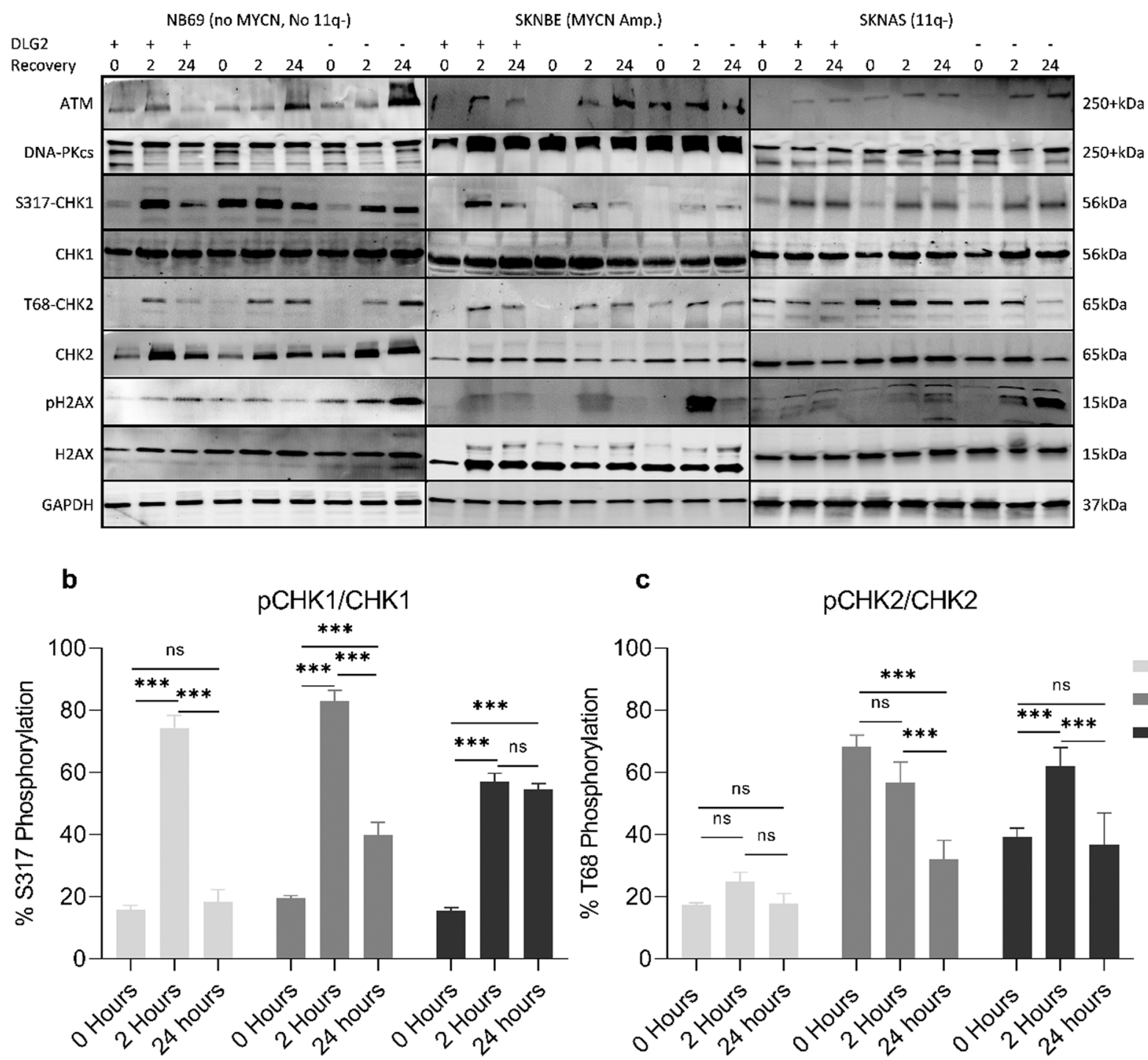

C

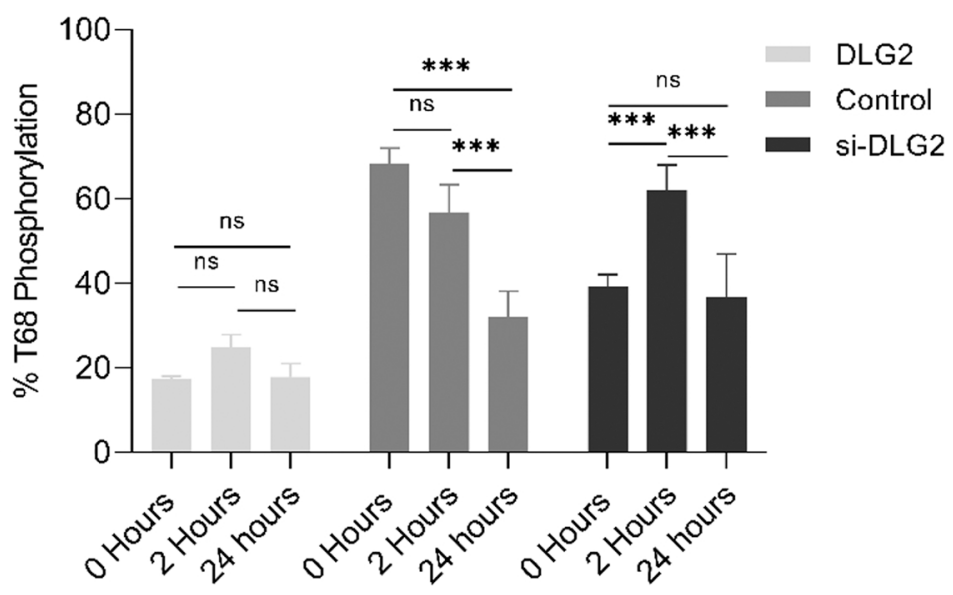

Fig. 5. NB cell lines with altered DLG2 expression show differential post translational modifications after UVC irradiation and recovery. a Protein expression patterns for CHK1-S317, CHK1, CHK2-T68, CHK2, ATM, DNA-PKCs, H2AX-S139, H2AX and the housekeeping gene GAPDH were determined by immunoblot before UVC, 0 h, as well as after $30 \mathrm{~s}$ UVC irradiation with either $2 \mathrm{~h}$ or $24 \mathrm{~h}$ recovery for DLG2 over expressed (+), mock transfected (Control) or DLG2 silenced (-), in NB69 (no MYCN-amplification or 11q-deletion), SKNBE (MYCN-amplified) and SKNAS (11q-deleted) cells. Quantification of the immunoblot by optical density showing the mean percentage of phosphorylation of (b) CHK1-S317 (CHK1-S317/total CHK1) and (c) CHK2-T68 (CHK2-T28/ total CHK2) across three experimental replicates for all three cell lines. The data in $\mathbf{b}$ and $\mathbf{c}$ are shown as the mean $\pm \mathrm{SD}$. The data shown are representative of three experimental replicates. ${ }^{*} P<0.05$, ${ }^{* *} \mathrm{P}<0.01$, ns $=$ not significant.

phosphorylation of p53 in response etoposide treatment increased in DLG2 silenced and control cells $(35.1 \%$ increased p53 phosphorylation compared to untreated, $p<0.001 \%$ and $6.7 \%$ increased p53 phosphorylation compared to untreated, $p<0.01$ ), however decreased after treatment in the DLG2 over expressed cells ( $42.7 \%$ less phosphorylation, $p<0.001$; Fig. 4i). The degree of S317- CHK1 phosphorylation was increased in all etoposide treated cells compared to untreated with the magnitude inverse to DLG2 expression with siDLG2, (54.9\% increase compared to untreated, $p<0.001)$ Control $(16.2 \%$ increase compared to untreated, $p<0.001)$ and DLG2 $(6.1 \%$ increase compared to untreated, $p<0.05$; Fig. 4j). The degree of T68-CHK2 phosphorylation was also markedly increased by etoposide treatment regardless of DLG2 expression level in SKNAS cells; siDLG2, (28.5\% increase compared to untreated, $p<0.001$ ) Control (73.6\% increase compared to untreated, $p<0.001)$ and DLG2 $(22.1 \%$ increase compared to untreated, $p<0.001$; Fig. $4 \mathrm{k}$ ), a response not seen in the SKNBE cells (Fig. $4 \mathrm{~g}$ ).

\subsection{High DLG2 levels enhanced CHK1 phosphorylation after UVC irradiation in NB cells}

To determine the effect of DLG2 on DNA repair in NB, we investigated DNA damage response pathways (DDR) in the NB cell lines NB69 ( $M Y C N$ and $11 \mathrm{q}$ normal), SKNBE (MYCN-amplified) and SKNAS (11q deleted) after DLG2 silencing or DLG2 over expression and UVC irradiation. We could show an increase in phosphorylated CHK1-S317 in all cells $2 \mathrm{~h}$ after UVC irradiation, with the lowest level in DLG2 silenced cells, in NB69 and SKNBE cells the degree of CHK1-phosphorylation was lowered after $24 \mathrm{~h}$ of recovery (Fig. 5a-b). There was no difference in basal CHK1 expression (Fig. 5a). Subsequent evaluation of CHK2-T68 phosphorylation demonstrated high basal expression in DLG2 silenced and control cells with equal phosphorylation after $2 \mathrm{~h}$ of recovery, decreasing after $24 \mathrm{~h}$ of recovery (Fig. 5a,c). No clear and consistent differences in the total amount of ATM or DNA-PKCs could be determined across the different cell lines. However, silenced DLG2 and $24 \mathrm{~h}$ 
recovery after UVC irradiation in NB69 showed produce an increase in ATM (Fig. 5a). To further investigate the presence of double-strand DNA breaks, we investigated the presence of $\gamma$-H2AX (Ser-139 phosphorylated H2AX, a biomarker of DNA double-strand breaks). We could determine that $\gamma$-H2AX levels were maintained at low levels in DLG2 over expression cells regardless of UVC exposure, indicating few remaining DNA breaks (Fig. 5a). $\gamma$-H2AX levels were increased after UVC exposure in all cells after DLG2 silencing, and at the highest level after $24 \mathrm{~h}$ of recovery, with the exception of SKNBE cells which showed a peek after $2 \mathrm{~h}$ (Fig. 5a).

We quantified the phosphorylation of S317 CHK1 and T68 CHK2 and could show that phosphorylation of CHK1-S317 increased from $0 \mathrm{~h}$ to two hours in all DLG2 transfections with the smallest magnitude observed in the DLG2 silenced cells ( $42 \%$ increased phosphorylation, $p<0.001)$ compared to the control and DLG2 over expressed cells (63.2\% increased phosphorylation, $p<0.001 \%$ and $59.7 \%$ increased phosphorylation, $p<0.001$; Fig. $5 \mathrm{~b}$ ). After 24 h recovery the $D L G 2$ over expressed cells were back to pre UVC treatment levels $(3.8 \%, \mathrm{p}=0.78)$ with the control and DLG2 silenced remaining at higher levels $(43.3 \%$ more phosphorylation compared to pre-treatment levels, $p<0.001 \%$ and $39.5 \%$ increased phosphorylation, $p<0.001$, respectively; Fig. $5 \mathrm{~b}$ ). The degree of phosphorylation of T68 CHK2 showed no significant differences following UVC exposure in the DLG2 over expressed cells. Between $2 \mathrm{~h}$ and $24 \mathrm{~h}$ recovery after UVC exposure the phosphorylation decreased in the DLG2 silenced and the control cells $(25.1 \%$ decrease between 2 and $24 \mathrm{~h}, p<0.001 \%$ and $24.57 \%$ decrease between 2 and 24 h, $p<0.001$; Fig. 5 c), with no difference in DLG2 over expressed cells.

\section{Discussion}

Previous attempts have been made to determine the illusive tumor suppressor gene located on 11q, with varying success [6]. It has previously been reported that there are two tumor suppressor genes located on chromosome $11 \mathrm{q}$ that could act in a combined haploinsufficient manner [27]. Here, we investigated the convergence of DLG2 (11q14.1) and the 11q23 deleted candidate TSGs within the DNA damage repair pathway. Previous studies have shown that some of the 11q23 SRO candidate TSGs function within this pathway [39-41], the role of DLG2 in DNA repair has only been previously suggested with bioinformatics [27]. We have shown changes in the expression of DNA repair genes in primary NB samples with unfavorable prognosis and 11q deletion (Fig. 1a-d). As patients with 11q deleted NBs are classified as high risk, with poor survival and a high number of DNA breaks and chromosomal alterations in the tumor, we investigated how DLG2 alters these genes of interest.

We have recently shown that there are two isoforms of DLG2 expressed in NB, isoform 2 (ISO2) and isoform 7 (ISO7); and that DLG2 isoform 7 is lost in advanced staged NB [42]. Here, we show that DLG2-ISO7 interrupts the expression of genes associated with the DNA damage repair machinery, i.e. PARP1, FEN1, PCNA as well as induce elevated MRE11a expression (Fig. 1e), another gene located in the 11q deleted region of NB. Inhibition of PARP1 [43,44], CHK1 [45] and PCNA [46] have previously been investigated in NB, while FEN1 is known to be up regulated in tumors with $M Y C N$ amplification [47]. We have previously shown that MYCN amplification results in lower DLG2 expression [27]. We now show that DLG2 represses FEN1 expression, potentially providing a mechanism as to why MYCN amplification results in increased FEN1 expression. To further investigate these findings, we silenced the fly orthologue gene $d m D L G$ in a Drosophila model, which subsequently resulted in increased $d m F E N$ and $d m P A R P$, thereby reproducing the altered PARP1 and FEN1 expression found in NB patient samples and NB cell lines (Fig. 1g). Subsequently we silenced $d m D L G$ in the fly and irradiated with UVC which resulted in increased DNA damage in the form of an increase in the number of sequences that could not be mapped to a reference sequence as well as the number of multiple mapped sequences, combined with an increased average read length compared to control, indicated that there were likely fusion-sequences present, indicative of low genome integrity (Fig. 2d and f). This was confirmed by looking at the number of multiple mapped sequences that were comprised of two distinct fragments (Fig. 2e). The loss of $d m D L G$ without the UVC treatment did not alter the number of fusions, however when combined with UVC irradiation activated low fidelity DNA repair; taken together with the results in human NB non-homologous end joining (NHEJ) reporter cells showing increased NHEJ after DLG2 silencing (Fig. 2c), we conclude that loss of $D L G$ results in increased NHEJ.

To further investigate the effects that DLG2 expression has on DNA repair, we induced DNA breaks by exposing transfected cells to UVC irradiation and allowed the cells to recover or exposed them to the double strand break inducing drug etoposide. Here we could show that DLG2 maintained genome stability and integrity, whereas loss of DLG2 resulted in high fragmentation even without the extra stimulus of the UVC irradiation or etoposide (Figs. 2a-b and Fig. 4a-b). This increase in DNA fragmentation may be the direct result of aberrantly activated endonuclease activity such as that found in FEN1 [48] or loss of the G2/M checkpoint. The increase in head size observed in the 11q deleted DLG2 overexpressed cells (Fig. 4a) may be attributed to maintenance of the cells at the G2/M checkpoint as previously established [27]. After either etoposide treatment or UVC stimulus, the NB cells with 11q-deletion or DLG2 silencing displayed a highly unstable genome with persisting DNA strand breaks. The 11q-deleted cells were not affected by $D L G 2$ silencing, likely since they already have very low expression of $D L G 2$. We have previously established that $D L G 2$ silencing reduces cell viability, implying a basal function [27] and correlating with observed clinical data. Over expression of DLG2 however seems effective in maintaining DNA-integrity after UV-exposure in both 11q-normal and 11q-deleted cells (Figs. 2a, 4a). To show that double-strand breaks were induced, we investigated phosphorylation of S139 H2AX. Here we could show that double-strand breaks were still present $24 \mathrm{~h}$ after UVC irradiation when DLG2 was down regulated (Fig. 5a), indicating that DNA repair had not yet occurred or that FEN1-mediated genome fragmentation was actively occurring. It has previously been shown that loss of DLG2 removes the G2/M DNA damage checkpoint [27], maintenance of which requires CHK1 activation [49]. Consistent with this, we here show that restoration of DLG2 expression produces a rapid S317 CHK1 phosphorylation, whereas there was a decrease and delay in phosphorylation in the DLG2 silenced cells (Fig. 5b) in response to UVC. Interestingly, there was a large increase in phosphorylated S317-CHK1 in the $D L G 2$ silenced cells after etoposide treatment (Fig. $4 \mathrm{f}$ and $\mathrm{j}$ ), not seen in the control or DLG2 over expressed cells despite the S139-H2AX marker present in all of the etoposide treated cells, and faintly present in the DLG2 over expressed untreated cells (Fig. 4c). The activation of S317 CHK1 occurs primarily through ATR but it can also occur through ATM [50]. The increasing phosphorylation of T68 CHK2 over time was present in the DLG2 silenced NB69 cells but not in the DLG2 over expressed cells. The phosphorylation of T68 CHK2 occurs primarily by ATM. Here we could show that ATM was highly expressed when $D L G 2$ was silenced in the 11q normal NB69 cells, indicating that ATM is operational when $D L G 2$ is inactive indicating that they function within different pathways [50].

We subsequently investigated the effects of $D L G 2$ on initiating apoptosis. We could show that elevated expression of $D L G 2$ resulted in increased total $\mathrm{p} 53$, the effect of which was seen in the expression of the transcriptional targets $B A X$ and $B C L 2$ (Fig. 3). The increased ratio of $\mathrm{BAX} / \mathrm{BCL} 2$ increases the permeability of the mitochondrial membrane as BCL2 cannot inhibit BAX pore formation and leads to apoptosis [51]. We could also detect an increase in pS46 phosphorylated p53 level, which is generally associated with severe genotoxic stress and leads to apoptosis [52]. Genotoxic stress is present in MYCN amplified NB tumors [43] as well as 11q deleted tumors [7]. We confirmed that there was a transient increase of the executioner caspases $3 / 7$ post DLG2 over expression, 
a

PARP1

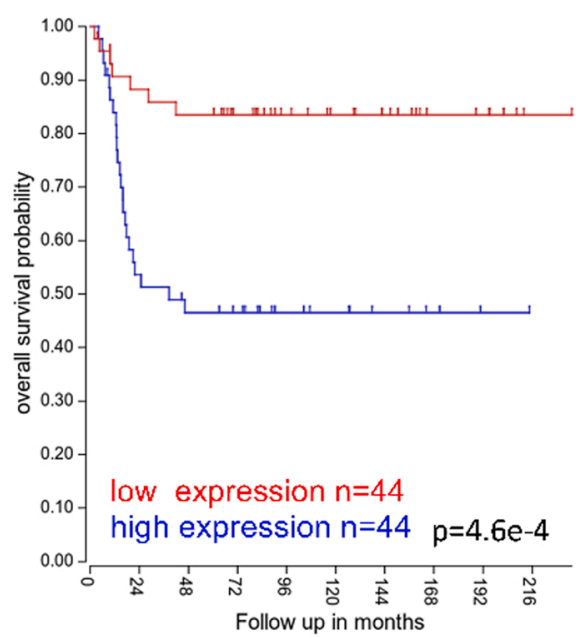

d

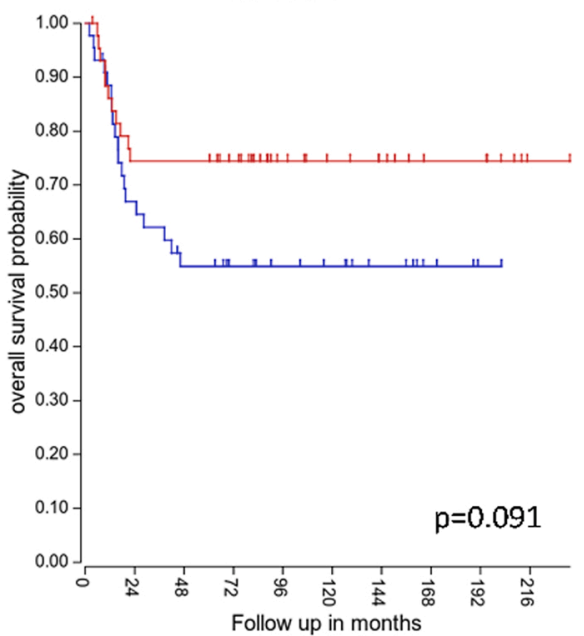

Follow up in months b

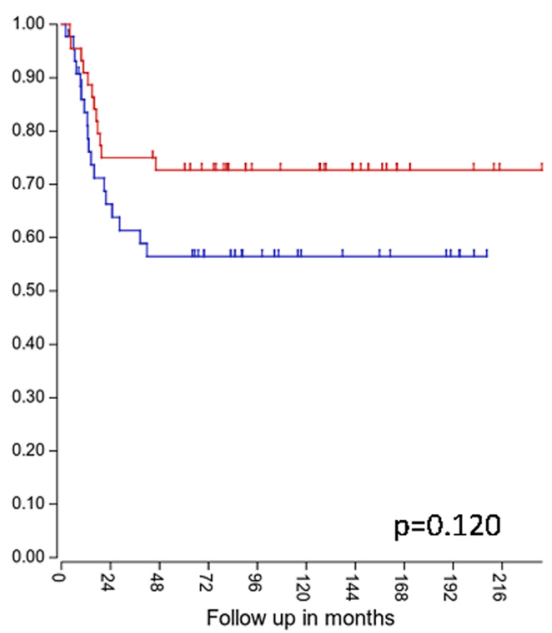

e

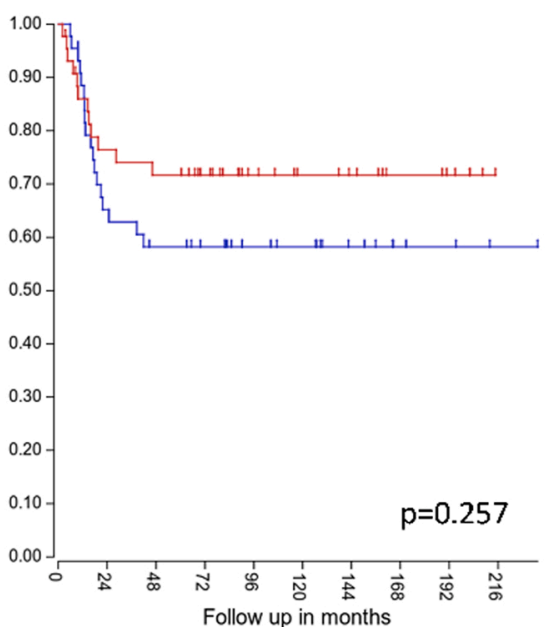

c

FEN1

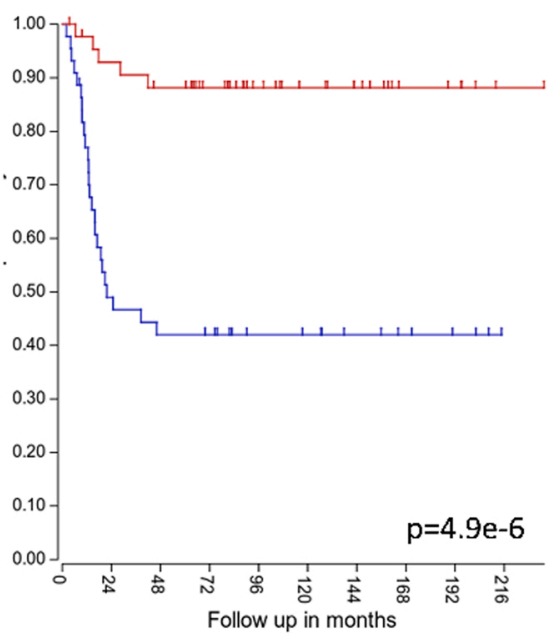

f

NBS1

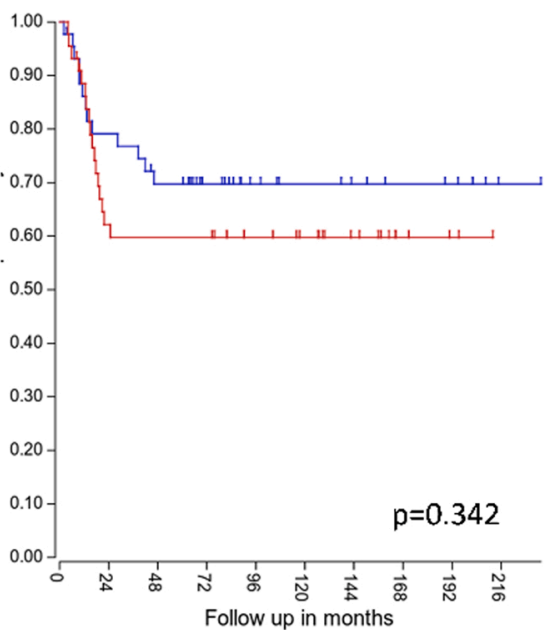

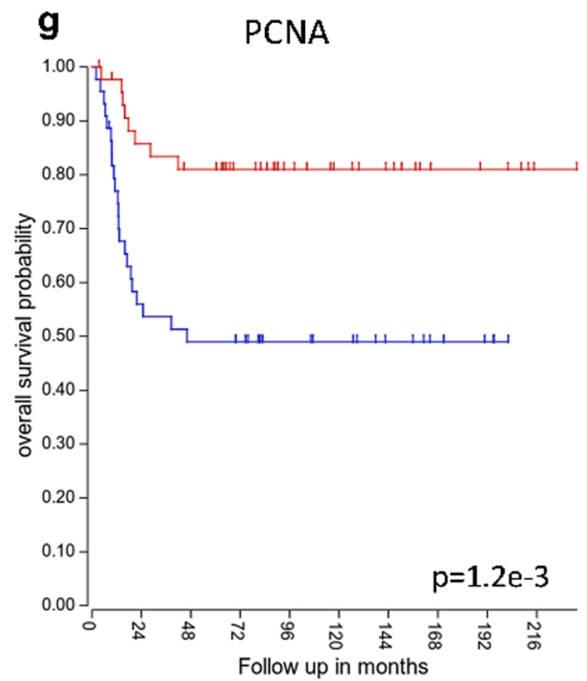

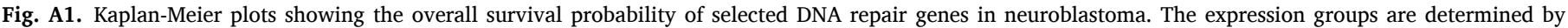

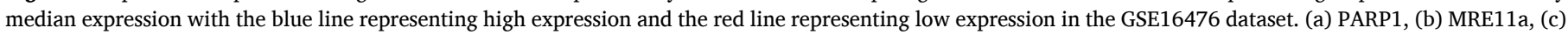
FEN1, (d) XRCC1, (e) LIG3, (f) NBS1 and (g) PCNA. Kaplan-Meier plots are determined using the product limit estimator followed by Bonferroni correction. 
showing that the pS46 p53 phosphorylation result in the initiation of apoptosis in NB by increasing BAX expression and allow for pS46 p53 mediated binding, a key step for transcription independent apoptosis [53]. However, the subsequent events of late apoptosis can be further assayed to confirm that apoptosis continues to completion and ultimately consolidate the results. ATM is one of the essential kinases that phosphorylates S46 p53 directly or indirectly $[23,40]$. In the data that we present $D L G 2$ and $A T M$ appear to be tentatively negatively correlated with low DLG2 increasing ATM protein expression in NB69, When combined with previous results showing that DLG2 is negatively associated with ATR [27], evidence is building that DLG2 prevents NHEJ DNA repair and results in cell death. Further studies into the coalescence of candidate $11 \mathrm{q}$ tumor suppressor genes are required to fully understand the causes of increased relapse and poor survival in $11 \mathrm{q}$ deleted NB patients.

\subsection{Conclusions}

We have shown that low DLG2 expression with additional DNA breakage stimulus results in high level of genome instability and increased NHEJ repair. DLG2 overexpression mediates the increase of pS46 p53 phosphorylation in response to genotoxicity in both 11q deleted and MYCN amplified NB cells, indicating that DLG2 can sustain the activation of apoptosis. This adds to the growing evidence that DLG2 is a NB tumor suppressor gene.

\section{CRediT authorship contribution statement}

Simon Keane: Conceptualization, Methodology, Validation, Formal analysis, Investigation, Resources, Data curation, Writing - original draft, Writing - review \& editing, Visualization. Hendrik A. de Weerd: Methodology, Validation, Formal analysis, Investigation, Data curation, Writing - review \& editing. Katarina Ejeskär: Conceptualization, Methodology, Formal analysis, Investigation, Data curation, Writing original draft, Writing - review \& editing, Visualization, Supervision, Project administration, Funding acquisition.

\section{Acknowledgements}

We thank the Swedish Childhood Cancer Fund [PR2016-0060], Jane and Dan Olsson Foundation [2020-29], Assar Gabrielssons Foundation [FB20-13], Nilsson-Ehle Endowments, Kungliga Fysiografiska sälskapet I Lund and University of Skövde for financial support.

\section{Appendix A}

See Fig. A1.

\section{References}

[1] T.J. Pugh, O. Morozova, E.F. Attiyeh, S. Asgharzadeh, J.S. Wei, D. Auclair, S L. Carter, K. Cibulskis, M. Hanna, A. Kiezun, et al., The genetic landscape of highrisk neuroblastoma, Nat. Genet. 45 (3) (2013) 279-284.

[2] K.R. Bosse, J.M. Maris, Advances in the translational genomics of neuroblastoma: From improving risk stratification and revealing novel biology to identifying actionable genomic alterations, Cancer 122 (1) (2016) 20-33.

[3] G.M. Brodeur, A.T. Look, H. Shimada, V.M. Hamilton, J.M. Maris, H.W. Hann, J. M. Leclerc, M. Bernstein, L.C. Brisson, J. Brossard, et al., Biological aspects of neuroblastomas identified by mass screening in Quebec, Med. Pediatr. Oncol. 36 (1) (2001) 157-159.

[4] J.M. Murphy, M.P. La Quaglia, Advances in the surgical treatment of neuroblastoma: a review, Eur. J. Pediatr. Surg. 24 (6) (2014) 450-456.

[5] K.A. Cole, J.M. Maris, New strategies in refractory and recurrent neuroblastoma: translational opportunities to impact patient outcome, Clin. Cancer Res. 18 (9) (2012) 2423-2428.

[6] V. Mlakar, S. Jurkovic Mlakar, G. Lopez, J.M. Maris, M. Ansari, F. Gumy-Pause, 11q deletion in neuroblastoma: a review of biological and clinical implications, Mol. Cancer 16 (1) (2017) 114.

[7] H. Caren, H. Kryh, M. Nethander, R.M. Sjoberg, C. Trager, S. Nilsson, J. Abrahamsson, P. Kogner, T. Martinsson, High-risk neuroblastoma tumors with 11q-deletion display a poor prognostic, chromosome instability phenotype with later onset, Proc. Natl. Acad. Sci. U. S. A. 107 (9) (2010) 4323-4328.

[8] P.S. White, P.M. Thompson, B.A. Seifried, E.P. Sulman, S.J. Jensen, C. Guo, J. M. Maris, M.D. Hogarty, C. Allen, J.A. Biegel, et al., Detailed molecular analysis of 1 p36 in neuroblastoma, Med. Pediatr. Oncol. 36 (1) (2001) 37-41.

[9] P.S. White, P.M. Thompson, T. Gotoh, E.R. Okawa, J. Igarashi, M. Kok, C. Winter, S.G. Gregory, M.D. Hogarty, J.M. Maris, et al., Definition and characterization of a region of 1 p36.3 consistently deleted in neuroblastoma, Oncogene 24 (16) (2005) 2684-2694.

[10] H. Caren, S. Fransson, K. Ejeskar, P. Kogner, T. Martinsson, Genetic and epigenetic changes in the common 1p36 deletion in neuroblastoma tumours, Br. J. Cancer 97 (10) (2007) 1416-1424.

[11] F. Abel, K. Ejeskar, P. Kogner, T. Martinsson, Gain of chromosome arm 17q is associated with unfavourable prognosis in neuroblastoma, but does not involve mutations in the somatostatin receptor 2(SSTR2) gene at 17q24, Br. J. Cancer 81 (8) (1999) 1402-1409.

[12] M. Morowitz, S. Shusterman, Y. Mosse, G. Hii, C.L. Winter, D. Khazi, Q. Wang, R. King, J.M. Maris, Detection of single-copy chromosome 17q gain in human neuroblastomas using real-time quantitative polymerase chain reaction, Mod. Pathol 16 (12) (2003) 1248-1256.

[13] C. Guo, P.S. White, M.J. Weiss, M.D. Hogarty, P.M. Thompson, D.O. Stram, R. Gerbing, K.K. Matthay, R.C. Seeger, G.M. Brodeur, et al., Allelic deletion at $11 q 23$ is common in MYCN single copy neuroblastomas, Oncogene 18 (35) (1999) $4948-4957$.

[14] J.M. Maris, C. Guo, P.S. White, M.D. Hogarty, P.M. Thompson, D.O. Stram, R. Gerbing, K.K. Matthay, R.C. Seeger, G.M. Brodeur, Allelic deletion at chromosome bands 11q14-23 is common in neuroblastoma, Med. Pediatr. Oncol. 36 (1) (2001) 24-27.

[15] M. Schwab, Enhanced expression of the cellular oncogene MYCN and progression of human neuroblastoma, Adv. Enzyme Regul. 31 (1991) 329-338.

[16] R. Corvi, L. Savelyeva, S. Breit, A. Wenzel, R. Handgretinger, J. Barak, M. Oren, L. Amler, M. Schwab, Non-syntenic amplification of MDM2 and MYCN in human neuroblastoma, Oncogene 10 (6) (1995) 1081-1086.

[17] J.M. Maris, How does MYCN amplification make neuroblastomas behave aggressively? Still more questions than answers, Pediatr. Blood Cancer 45 (7) (2005) 869-870.

[18] J. Buechner, E. Tomte, B.H. Haug, J.R. Henriksen, C. Lokke, T. Flaegstad, C. Einvik, Tumour-suppressor microRNAs let-7 and mir-101 target the proto-oncogene MYCN and inhibit cell proliferation in MYCN-amplified neuroblastoma, Br. J. Cancer 105 (2) (2011) 296-303.

[19] H.H. Chang, M.Y. Lu, Y.L. Yang, S.W. Chou, D.T. Lin, K.H. Lin, W.M. Hsu, Y. M. Jeng, S.T. Jou, The prognostic roles of and correlation between ALK and MYCN protein expression in neuroblastoma, J. Clin. Pathol. 73 (3) (2020) 154-161.

[20] A. Shibata, P. Jeggo, M. Lobrich, The pendulum of the Ku-Ku clock, DNA Repair ((Amst)) 71 (2018) 164-171.

[21] C. Ma, K. Ha, M.S. Kim, Y.W. Noh, H. Lin, L. Tang, Q. Zhu, D. Zhang, H. Chen, S. Han, et al., The anaphase promoting complex promotes NHEJ repair through stabilizing Ku80 at DNA damage sites, Cell Cycle 17 (9) (2018) 1138-1145.

[22] Y.F. Lin, H.Y. Shih, Z.F. Shang, C.T. Kuo, J. Guo, C. Du, H. Lee, B.P.C. Chen, PIDD mediates the association of DNA-PKcs and ATR at stalled replication forks to facilitate the ATR signaling pathway, Nucleic Acids Res. 46 (4) (2018) 1847-1859.

[23] A.K. Ashley, C.J. Kemp, DNA-PK, ATM, and ATR: PIKKing on p53, Cell Cycle 17 (3) (2018) 275-276.

[24] S. Sharma, S.M. Javadekar, M. Pandey, M. Srivastava, R. Kumari, S.C. Raghavan, Homology and enzymatic requirements of microhomology-dependent alternative end joining, Cell Death Dis. 6 (2015), e1697.

[25] B.J. Eckelmann, A. Bacolla, H. Wang, Z. Ye, E.N. Guerrero, W. Jiang, R. El-Zein, M. L. Hegde, A.E. Tomkinson, J.A. Tainer, et al., XRCC1 promotes replication restart, nascent fork degradation and mutagenic DNA repair in BRCA2-deficient cells, NAR Cancer 2 (3) (2020) zcaa013.

[26] S. Ahrabi, S. Sarkar, S.X. Pfister, G. Pirovano, G.S. Higgins, A.C. Porter, T. C. Humphrey, A role for human homologous recombination factors in suppressing microhomology-mediated end joining, Nucleic Acids Res. 44 (12) (2016) 5743-5757.

[27] S. Keane, S. Ameen, A. Lindlof, K. Ejeskar, Low DLG2 gene expression, a link between 11q-deleted and MYCN-amplified neuroblastoma, causes forced cell cycle progression, and predicts poor patient survival, Cell Commun. Signal. 18 (1) (2020) 65 .

[28] J.T. Siaw, N. Javanmardi, J.Vd Eynden, D.E. Lind, S. Fransson, A. MartinezMonleon, A. Djos, R.M. Sjoberg, M. Ostensson, H. Caren, et al., 11q Deletion or ALK Activity Curbs DLG2 Expression to Maintain an Undifferentiated State in Neuroblastoma, Cell Rep. 32 (12) (2020), 108171.

[29] Y.W. Shao, G.A. Wood, J. Lu, Q.L. Tang, J. Liu, S. Molyneux, Y. Chen, H. Fang, H. Adissu, T. McKee, et al., Cross-species genomics identifies DLG2 as a tumor suppressor in osteosarcoma, Oncogene 38 (2) (2019) 291-298.

[30] R.J. Zhuang, X.X. Bai, W. Liu, MicroRNA-23a depletion promotes apoptosis of ovarian cancer stem cell and inhibits cell migration by targeting DLG2, Cancer Biol. Ther. (2019) 1-15.

[31] C. Wang, B. Gong, P.R. Bushel, J. Thierry-Mieg, D. Thierry-Mieg, J. Xu, H. Fang H. Hong, J. Shen, Z. Su, et al., The concordance between RNA-seq and microarray data depends on chemical treatment and transcript abundance, Nat. Biotechnol. 32 (9) (2014) 926-932.

[32] J.J. Molenaar, J. Koster, D.A. Zwijnenburg, P. van Sluis, L.J. Valentijn, I. van der Ploeg, M. Hamdi, J. van Nes, B.A. Westerman, J. van Arkel, et al., Sequencing of neuroblastoma identifies chromothripsis and defects in neuritogenesis genes, Nature 483 (7391) (2012) 589-593. 
[33] Q. Wang, S. Diskin, E. Rappaport, E. Attiyeh, Y. Mosse, D. Shue, E. Seiser, J. Jagannathan, S. Shusterman, M. Bansal, et al., Integrative genomics identifies distinct molecular classes of neuroblastoma and shows that multiple genes are targeted by regional alterations in DNA copy number, Cancer Res. 66 (12) (2006) 6050-6062.

[34] K.O. Henrich, S. Bender, M. Saadati, D. Dreidax, M. Gartlgruber, C. Shao, C. Herrmann, M. Wiesenfarth, M. Parzonka, L. Wehrmann, et al., Integrative genome-scale analysis identifies epigenetic mechanisms of transcriptional deregulation in unfavorable neuroblastomas, Cancer Res. 76 (18) (2016) 5523-5537.

[35] K.J. Livak, T.D. Schmittgen, Analysis of relative gene expression data using realtime quantitative PCR and the 2(-Delta Delta C(T)) Method, Methods 25 (4) (2001) $402-408$.

[36] T.D. Schmittgen, K.J. Livak, Analyzing real-time PCR data by the comparative C(T) method, Nat. Protoc. 3 (6) (2008) 1101-1108.

[37] A. Larkin, S.J. Marygold, G. Antonazzo, H. Attrill, G. Dos Santos, P.V. Garapati, J. L. Goodman, L.S. Gramates, G. Millburn, V.B. Strelets, et al., FlyBase: updates to the Drosophila melanogaster knowledge base, Nucleic Acids Res. 49 (D1) (2021) D899-D907.

[38] P.J.A. Cock, T. Antao, J.T. Chang, B.A. Chapman, C.J. Cox, A. Dalke, I. Friedberg, T. Hamelryck, F. Kauff, B. Wilczynski, et al., Biopython: freely available Python tools for computational molecular biology and bioinformatics, Bioinformatics 25 (11) (2009) 1422-1423.

[39] Y. Li, H. Xiong, D.Q. Yang, Functional switching of ATM: sensor of DNA damage in proliferating cells and mediator of Akt survival signal in post-mitotic human neuron-like cells, Chin. J. Cancer 31 (8) (2012) 364-372.

[40] M. Katsuyama, K. Iwata, M. Ibi, K. Matsuno, M. Matsumoto, C. Yabe-Nishimura, Clioquinol induces DNA double-strand breaks, activation of ATM, and subsequent activation of p53 signaling, Toxicology 299 (1) (2012) 55-59.

[41] S.J. Mandriota, L.J. Valentijn, L. Lesne, D.R. Betts, D. Marino, M. BoudalKhoshbeen, W.B. London, A.L. Rougemont, E.F. Attiyeh, J.M. Maris, et al., Ataxiatelangiectasia mutated (ATM) silencing promotes neuroblastoma progression through a MYCN independent mechanism, Oncotarget 6 (21) (2015) $18558-18576$.

[42] S. Keane, T. Martinsson, P. Kogner, K. Ejeskar, The loss of DLG2 isoform 7/8, but not isoform 2, is critical in advanced staged neuroblastoma, Cancer Cell Int. 21 (1) (2021) 170.
[43] D. King, X.D. Li, G.S. Almeida, C. Kwok, P. Gravells, D. Harrison, S. Burke, A. Hallsworth, Y. Jamin, S. George, et al., MYCN expression induces replication stress and sensitivity to PARP inhibition in neuroblastoma, Oncotarget 11 (23) (2020) 2141-2159.

[44] E. Sanmartin, L. Munoz, M. Piqueras, J.A. Sirerol, P. Berlanga, A. Canete, V. Castel, Font, J. de Mora, Deletion of $11 \mathrm{q}$ in Neuroblastomas Drives Sensitivity to PARP Inhibition, Clin. Cancer Res. 23 (22) (2017) 6875-6887.

[45] S. Di Giulio, V. Colicchia, F. Pastorino, F. Pedretti, F. Fabretti, V. Nicolis di Robilant, V. Ramponi, G. Scafetta, M. Moretti, V. Licursi, et al., A combination of PARP and CHK1 inhibitors efficiently antagonizes MYCN-driven tumors, Oncogene 40 (43) (2021) 6143-6152.

[46] L. Gu, S. Smith, C. Li, R.J. Hickey, J.M. Stark, G.B. Fields, W.H. Lang, J. A. Sandoval, L.H. Malkas, A PCNA-derived cell permeable peptide selectively inhibits neuroblastoma cell growth, PLoS One 9 (4) (2014), e94773.

[47] A. Krause, V. Combaret, I. Iacono, B. Lacroix, C. Compagnon, C. Bergeron, S. Valsesia-Wittmann, P. Leissner, B. Mougin, A. Puisieux, Genome-wide analysis of gene expression in neuroblastomas detected by mass screening, Cancer Lett. 225 (1) (2005) 111-120.

[48] L. Zheng, J. Jia, L.D. Finger, Z. Guo, C. Zer, B. Shen, Functional regulation of FEN1 nuclease and its link to cancer, Nucleic Acids Res. 39 (3) (2011) 781-794.

[49] K. Ando, Y. Nakamura, H. Nagase, A. Nakagawara, T. Koshinaga, S. Wada, M. Makishima, Co-Inhibition of the DNA Damage Response and CHK1 Enhances Apoptosis of Neuroblastoma Cells, Int. J. Mol. Sci. 20 (15) (2019).

[50] J. Bartek, J. Lukas, Chk1 and Chk2 kinases in checkpoint control and cancer, Cancer Cell 3 (5) (2003) 421-429.

[51] Q. Wang, L. Zhang, X. Yuan, Y. Ou, X. Zhu, Z. Cheng, P. Zhang, X. Wu, Y. Meng, L. Zhang, The Relationship between the Bcl-2/Bax Proteins and the MitochondriaMediated Apoptosis Pathway in the Differentiation of Adipose-Derived Stromal Cells into Neurons, PLoS One 11 (10) (2016), e0163327.

[52] M. Petroni, V. Veschi, A. Prodosmo, C. Rinaldo, I. Massimi, M. Carbonari, C. Dominici, H.P. McDowell, C. Rinaldi, I. Screpanti, et al., MYCN sensitizes human neuroblastoma to apoptosis by HIPK2 activation through a DNA damage response, Mol Cancer Res. 9 (1) (2011) 67-77.

[53] A.V. Follis, F. Llambi, P. Merritt, J.E. Chipuk, D.R. Green, R.W. Kriwacki, Pin1Induced Proline Isomerization in Cytosolic p53 Mediates BAX Activation and Apoptosis, Mol. Cell 59 (4) (2015) 677-684. 